\title{
Synthesis, Structure, Spectral and Anion Sensing Studies of Aromatic Meso-Fused Boron(III) Benzitriphyrin(2.1.1) Complex
}

Avisikta Sinhas ${ }^{\S}$ Ankit Kumar ${ }^{\S}$ and Mangalampalli Ravikanth*

Department of Chemistry, Indian Institute of Technology Bombay, Powai, Mumbai 400076, India, E-mail: ravikanth@chem.iitb.ac.in

\begin{tabular}{|c|c|c|}
\hline Sr. no & Details & Page no. \\
\hline 1 & General Experimental Section & S2-S5 \\
\hline 2 & $\begin{array}{l}\text { Figures S1-S7. Characterization (HRMS and NMR) data for } \\
\text { compound } \mathbf{1 0 .}\end{array}$ & S6-S12 \\
\hline 3 & $\begin{array}{l}\text { Figure S8, Table S4-S5, Figure S9. X-ray data, Crystal packing } \\
\text { diagram of Compound } 10\end{array}$ & S13-S17 \\
\hline 4 & $\begin{array}{l}\text { Figures S10-S12. DFT optimized structure, NICS and HOMA } \\
\text { indices of Compound } \mathbf{1 0}\end{array}$ & S18-S19 \\
\hline 5 & $\begin{array}{l}\text { Figures S13-S14. Color change and comparison of absorption, } \\
\text { emission spectra of Compound } \mathbf{1 0} \text { in the presence of various anions }\end{array}$ & S20 \\
\hline 6 & 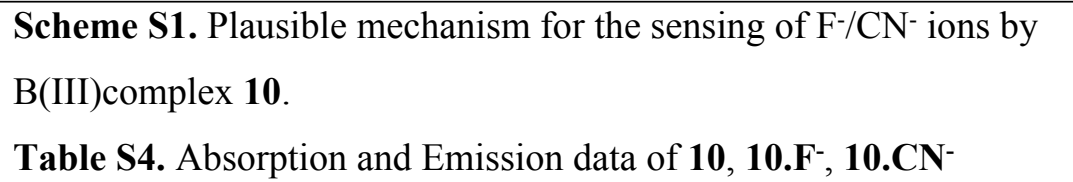 & S21 \\
\hline 7 & $\begin{array}{l}\text { Figure S15. The absorption spectra and emission spectra of } \\
\text { Compound } \mathbf{1 0} \text { in the presence of } \mathrm{F}^{-} \text {and } \mathrm{CN}^{-} \text {ions }\end{array}$ & S22 \\
\hline 8 & $\begin{array}{l}\text { Figure S16-S17. Job's Plot of Compound } \mathbf{1 0} \text { in the presence of } \mathrm{F}^{-} \\
\text {and } \mathrm{CN}^{-} \text {ions }\end{array}$ & S23-S24 \\
\hline 9 & 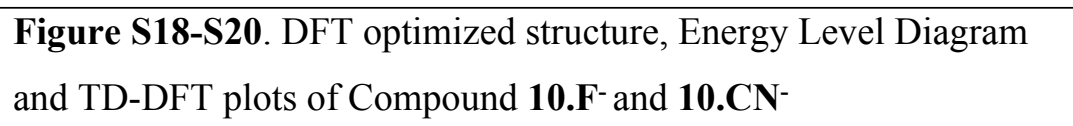 & S25-S26 \\
\hline 10 & 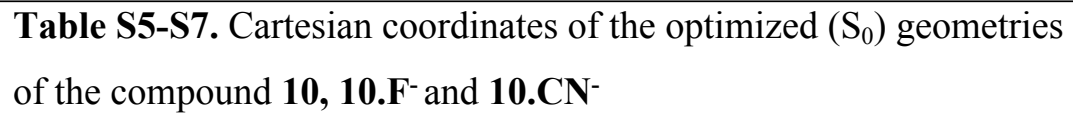 & S27-S29 \\
\hline 11 & Table S8-S10. Selected TD-DFT calculated oscillator strengths and & $\mathrm{S} 30-\mathrm{S} 32$ \\
\hline
\end{tabular}




\begin{tabular}{|c|l|c|}
\hline & $\begin{array}{l}\text { compositions of the major electronic transitions of } \mathbf{1 0}, \mathbf{1 0 . F ^ { - }} \text { and } \\
\mathbf{1 0 . C N ^ { - }}\end{array}$ & \\
\hline 12 & References & $\mathrm{S} 33$ \\
\hline
\end{tabular}




\section{General Experimental Section}

Reagent grade chemicals were employed during synthesis. For column chromatography purification purpose, silica (60-120 and 100-200 mesh), basic alumina and neutral alumina was used. Bruker 400 and $500 \mathrm{MHz}$ instruments assisted us in recording $1 \mathrm{D}, 2 \mathrm{D},{ }^{13} \mathrm{C},{ }^{11} \mathrm{~B}$ and ${ }^{19} \mathrm{~F}$ using $\mathrm{CDCl}_{3}$ as solvent. 100 and $125.7 \mathrm{MHz}$ is the frequency for the ${ }^{13} \mathrm{C}$ nucleus whereas for ${ }^{11} \mathrm{~B}$ and ${ }^{19} \mathrm{~F}$ nucleus the frequency is 160 and $471 \mathrm{MHz}$ for $500 \mathrm{MHz}$ instruments respectively. For ${ }^{1} \mathrm{H}$ and ${ }^{13} \mathrm{C}$ NMR the internal standard used was Tetramethylsilane $\left[\mathrm{Si}\left(\mathrm{CH}_{3}\right)_{4}\right]$. Cary series UV-vis-NIR and UV 3600 Shimadzu spectrophotometer helped us to record absorption spectra of the compounds. BAS electrochemical system was used for carrying out Cyclic voltammetry (CV) studies making use of the three-electrode configuration comprising of glassy carbon (working electrode), saturated calomel (reference electrode) electrodes and platinum wire (auxiliary electrode). Experiments were done in dry dichloromethane using $0.1 \mathrm{M}$ tetrabutylammonium perchlorate as a supporting electrolyte. Half wave potentials were measured using DPV and also calculated manually by taking the average of cathodic and anodic peak potentials. Before performing the spectral and electrochemical measurements, all the solutions were purged with argon gas. HRMS was recorded on a Bruker maXis Impact and Q-Tof micro mass spectrometer using positive mode ESI methods for acetonitrile/methanol solutions. For UV-vis stock solution of compound $\mathbf{1 0}\left(1 \times 10^{-5} \mathrm{M}\right)$ was prepared by using a HPLC grade $\mathrm{CHCl}_{3}$ solvent.

X-ray Crystal Structure Analysis. Rigaku Saturn 724 diffractometer was used for performing Single-crystal X-ray structure analysis which comprised of a low-temperature attachment. Data were collected at $100 \mathrm{~K}$ using graphite-monochromated Mo K $\alpha$ radiation $(\lambda \alpha=0.71073 \AA)$ by the $\omega$-scan technique. Data were reduced by using Crystal Clear-SM Expert 2.1 b24 software. 
Structures were solved by direct methods and refined by least-squares against F2 utilizing the software packages SHELXL-97,30 SIR-92,31 and WINGX.32 All nonhydrogen atoms were refined anisotropically. X-ray data for the Boron complex of meso-fused benzitriphyrin(2.1.1) 10 were collected on a Bruker Kappa CCD diffractometer equipped with a graphite monochromated Mo Ka radiation source at $200 \mathrm{~K}$ using the $\theta-2 \theta$ scan mode. An empirical absorption correction by multi scans was applied and all of the non-hydrogen atoms were refined with anisotropic displacement factors. Hydrogen atoms were placed in ideal positions and fixed with relative isotropic displacement parameters. Solvent molecules that could not be identified or modeled were found and eventually squeezed using PLATON.17. The corresponding loop of the residual electron-voids (from PLATON) was appended in the corresponding CIF file. Single crystals for $\mathrm{X}$-ray analysis were grown by slow evaporation of compound $\mathbf{1 0}$ in $n$-hexane/chloroform solution for 4-5 days. CCDC no. 2106226 contains supplementary crystallographic data for this paper. These data can be obtained free of charge from The Cambridge Crystallographic Data Centre via www.ccdc.cam.ac.uk/data_request/cif or by emailing data_request@ccdc.cam.ac.uk, or by contacting The Cambridge Crystallographic Data Centre, 12 Union Road, Cambridge CB2 1EZ, UK; fax: +44 1223336033 .

Details of Anion Binding Studies. Tetrabutylammonium salts and analytical grade solvents were used during titration for anion binding studies. Stock solutions of the anions used were tetrabutylammonium cyanide (TBACN, $3 \times 10^{-5} \mathrm{M}$ ), tetrabutylammonium fluoride (TBAF, 3X10$\left.{ }^{5} \mathrm{M}\right)$ and a stock solution of $\mathbf{1 0}\left(3 \times 10^{-4} \mathrm{M}\right)$ were prepared in $\mathrm{CHCl}_{3}$. Systematic titration experiments were carried out by diluting a solution of $\mathbf{1 0}$ and keeping $3 \mathrm{~mL}$ of solution $\mathbf{1 0}$ (10 $\mu \mathrm{M})$ in a quartz cuvette of $1 \mathrm{~cm}$ path length and varying quantity of anions were added incrementally by employing a micropipette. For fluorescence measurements, excitation was 
provided at $420 \mathrm{~nm}$, and emission was collected from 450 to $850 \mathrm{~nm}$. Association constant of the anion complex has been calculated by using the standard where $\mathrm{I}_{0}$ is the intensity of $\mathbf{1 0}$ before the addition of anion, $\mathrm{I}$ is the intensity in the presence of $\mathrm{A}, \mathrm{I}_{1}$ is intensity upon saturation with $\mathrm{A}$, and $\mathrm{Ka}$ is the association constant of complex formed.

$$
\frac{1}{I-I_{0}}=\frac{1}{I_{I^{-}} I_{0}}+\frac{1}{\left(I_{I^{-}}-I_{0}\right) K_{a}\left[A^{-}\right]}
$$

Computational Details. Gaussian 09 program package $^{1}$ was used for performing all the calculations. The structures of compounds $10,10 . F^{-}$and $\mathbf{1 0 . C N}-$ in ground $\left(\mathrm{S}_{0}\right)$ states were optimized using The density functional theory (DFT) ${ }^{2}$ method, hybrid functional B3LYP in conjunction with basis set 6-31G(d,p). ${ }^{3}$ Vertical excitation energies were calculated from TDDFT techniques for $\mathrm{S}_{0} \rightarrow \mathrm{Sn}$ transitions, to obtain the oscillator strengths, an identical basis and functional hybrid set were used. ${ }^{4}$ All the computations were calculated under the Polarisable Continuum Model $(\mathrm{PCM})^{5}$ in the $\mathrm{CHCl}_{3}$ media using the Self-Consistent Reaction Field (SCRF). The electronic absorption spectra, as well as the oscillator strengths, were thoroughly examined using TD-DFT with PCM model based on the optimized structures in the $\mathrm{S}_{0}$ state. 


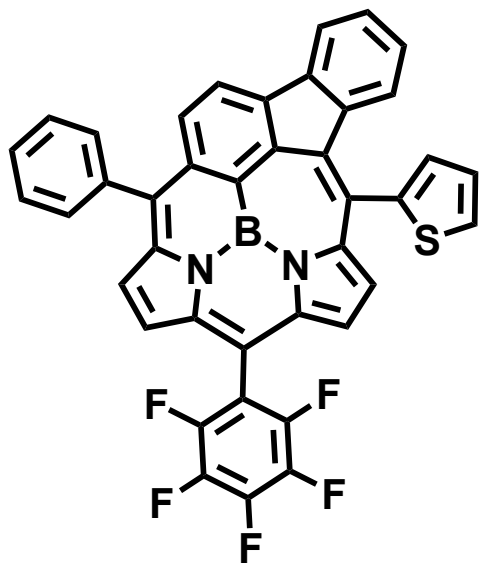

Compound 10

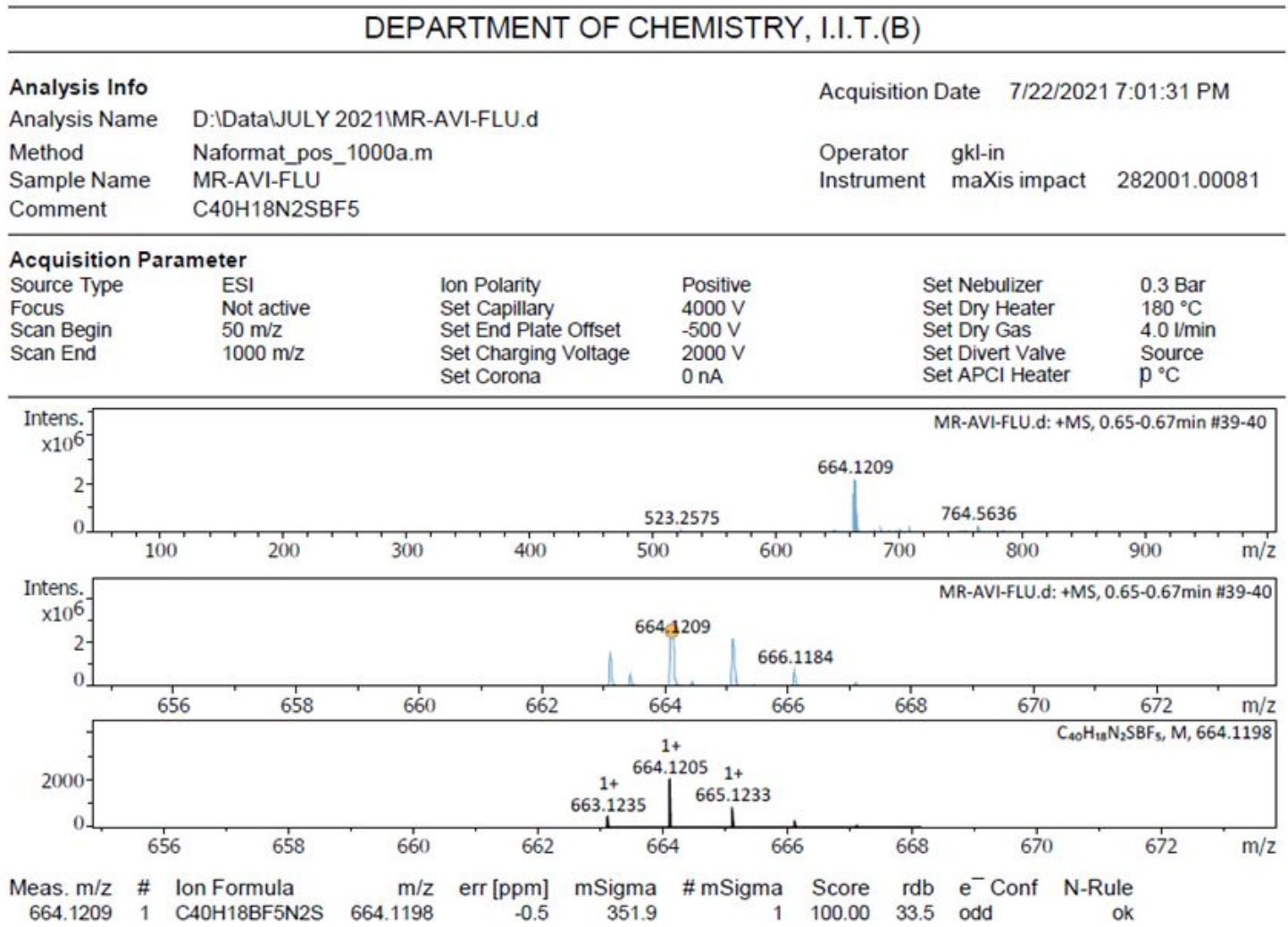

Figure S1. HR mass spectrum of the compound $\mathbf{1 0 .}$ 


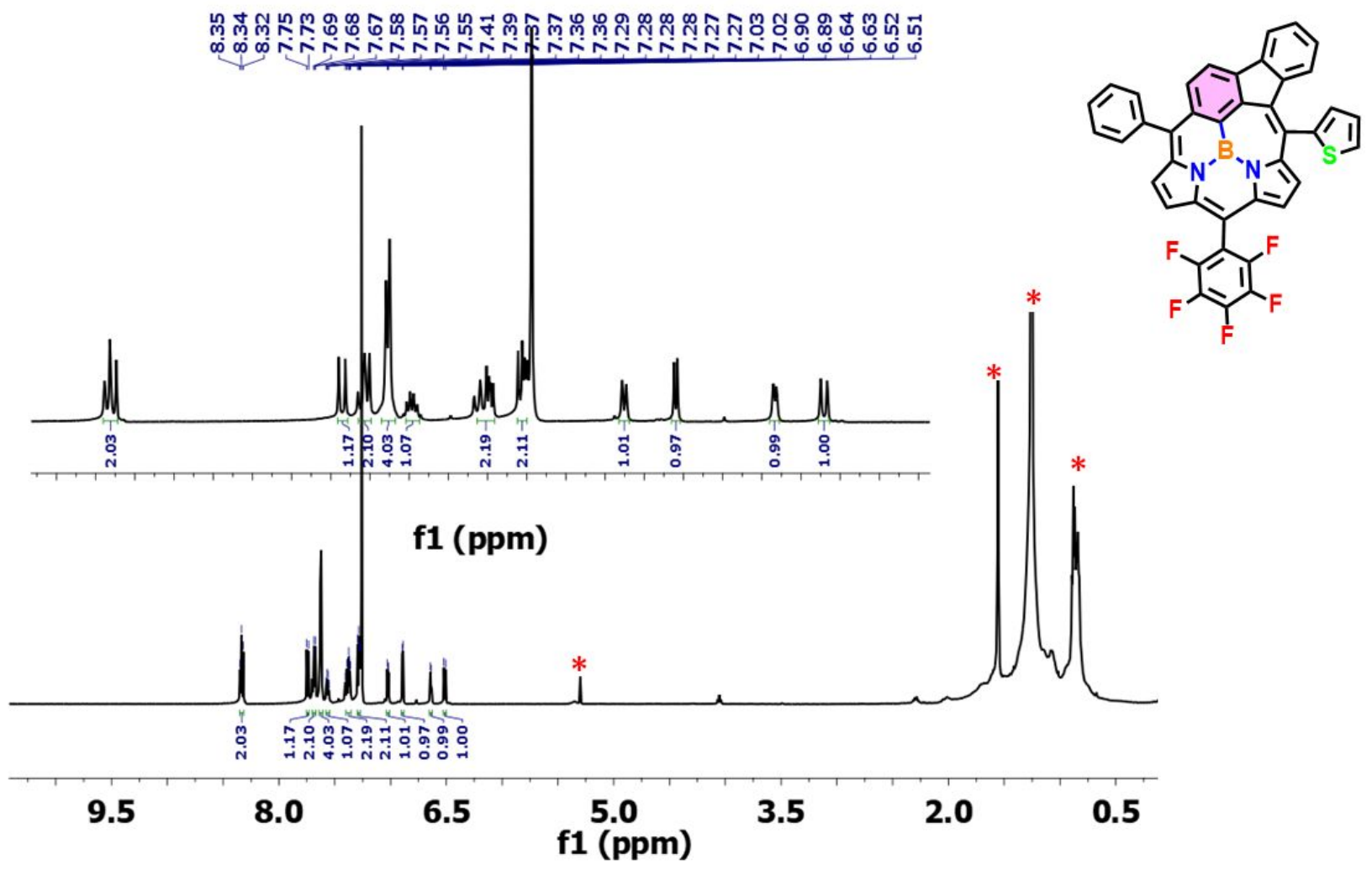

Figure S2. ${ }^{1} \mathrm{H}$ NMR spectrum of the compound 10 recorded in $\mathrm{CDCl}_{3}$ on $500 \mathrm{MHz} \mathrm{NMR}$ instrument. Note: Peaks marked with asterisk $(*)$ are due to residual solvents 


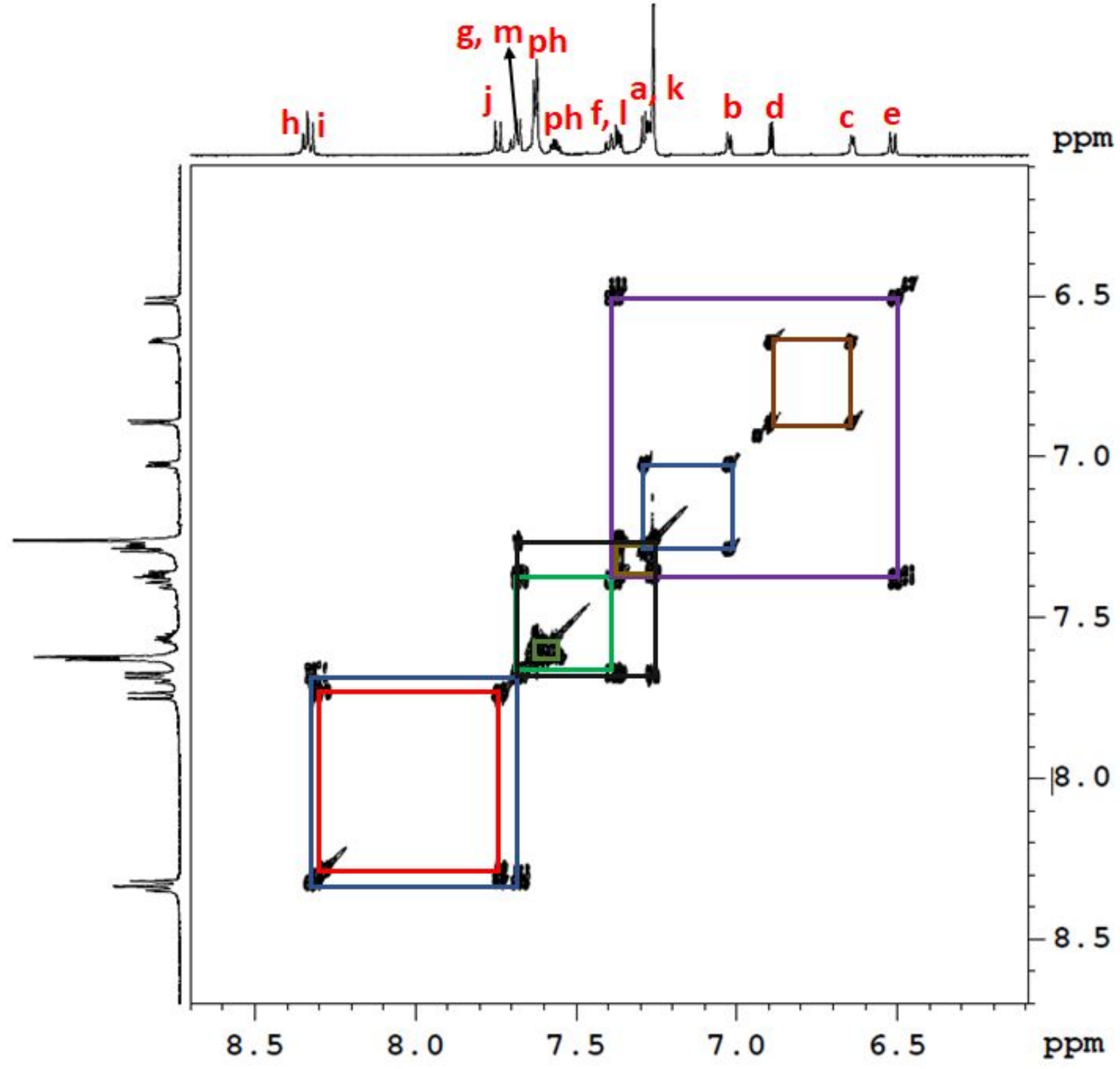

Figure S3. ${ }^{1} \mathrm{H}-{ }^{1} \mathrm{H}$ COSY of Compound $\mathbf{1 0}$ recorded in $\mathrm{CDCl}_{3}$. 


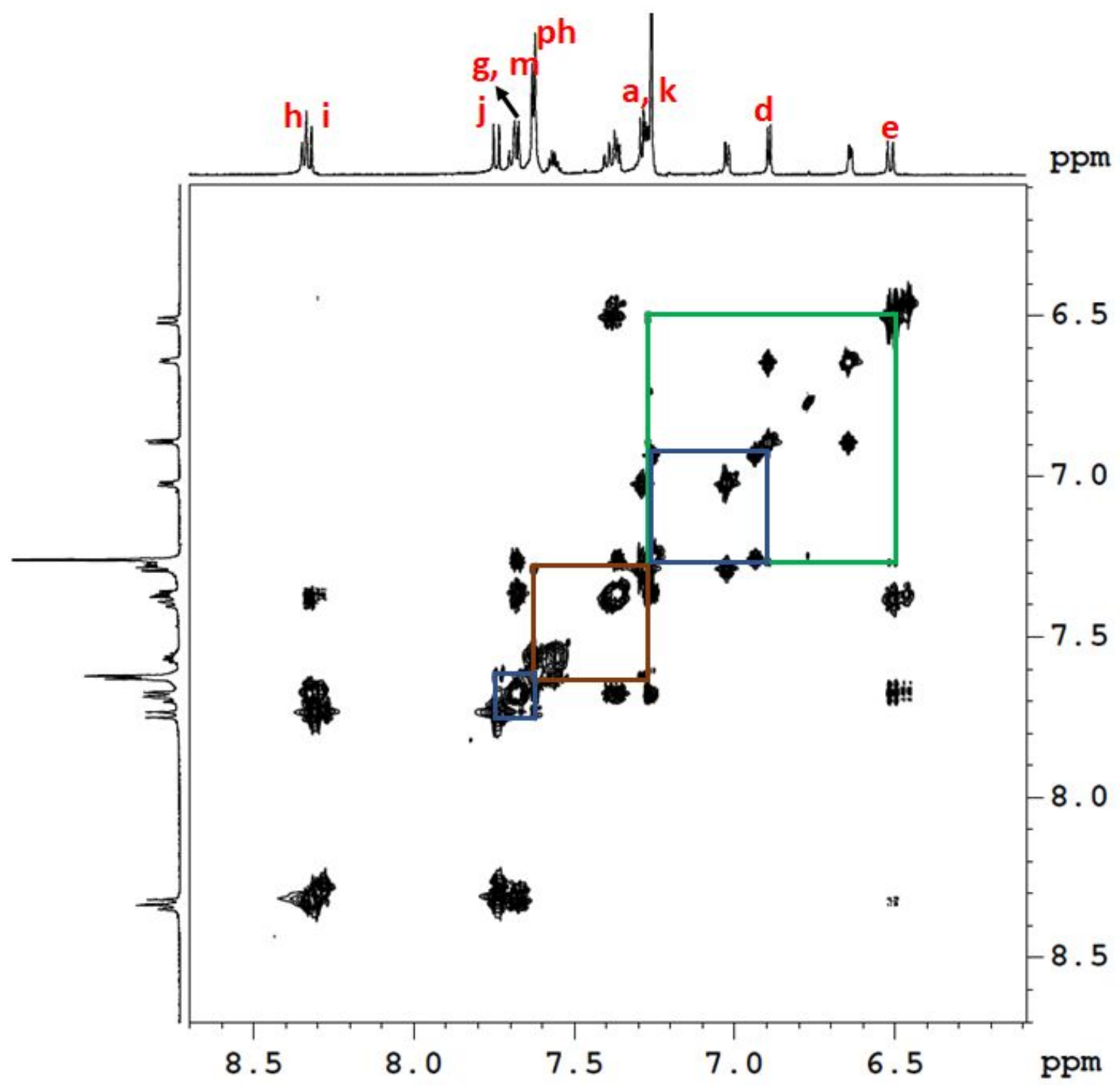

Figure S4. ${ }^{1} \mathrm{H}-{ }^{1} \mathrm{H}$ NOESY of Compound 10 recorded in $\mathrm{CDCl}_{3}$. 


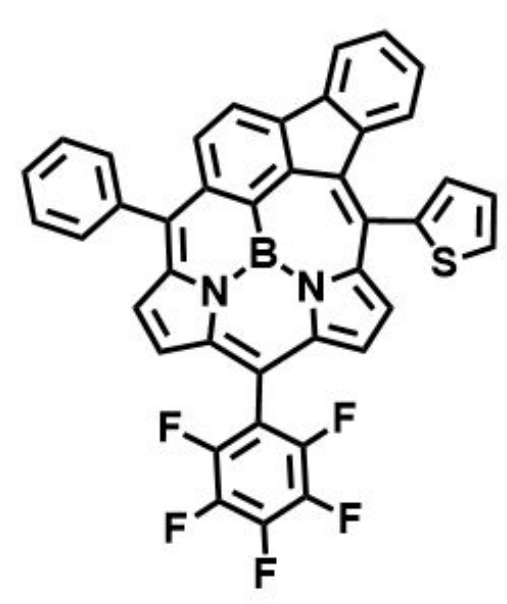

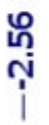

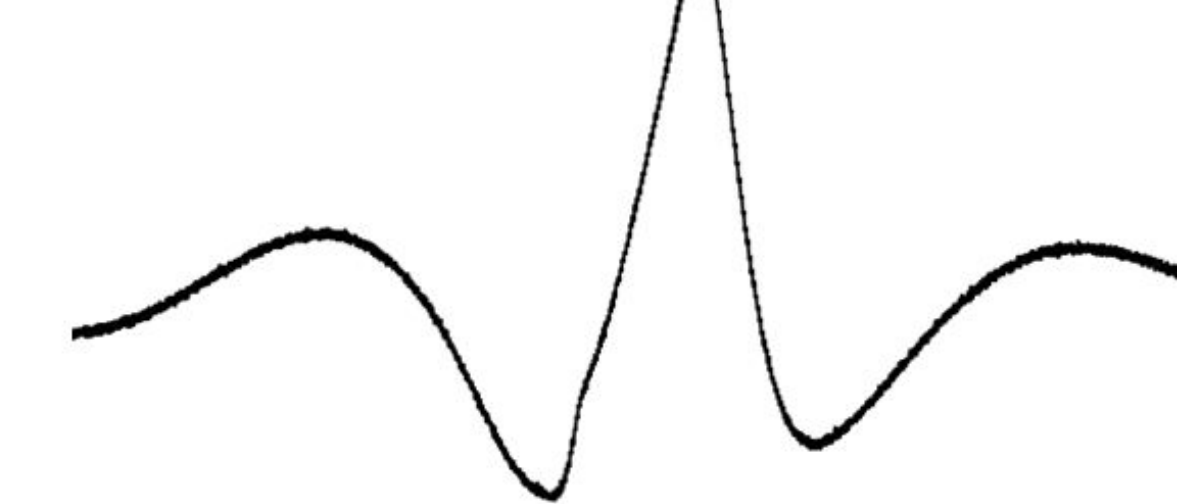

\begin{tabular}{|c|c|c|c|c|c|}
\hline 120 & 80 & 40 & $\stackrel{0}{\text { f1 (ppm) }}$ & -40 & -80 \\
\hline
\end{tabular}

Figure S5. ${ }^{11} \mathrm{~B}$ NMR of Compound 10 recorded in $\mathrm{CDCl}_{3}$ on $500 \mathrm{MHz}$ NMR instrument 


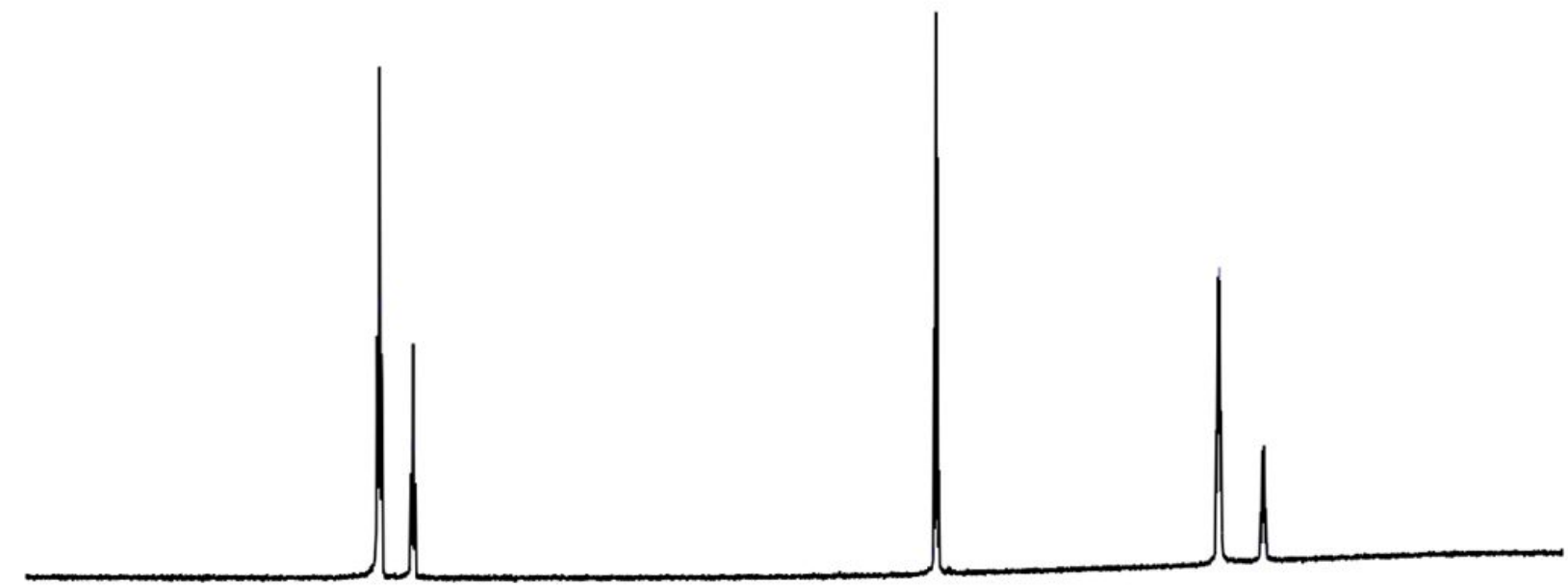

\begin{tabular}{llllllllll|l|l|l|}
\hline-128 & -132 & -136 & -140 & -144 & $\begin{array}{l}-148 \\
f 1(\mathrm{ppm})\end{array}$ & -152 & -156 & -160 & -164 & -168
\end{tabular}

Figure S6. ${ }^{19} \mathrm{~F}$ NMR of Compound 10 recorded in $\mathrm{CDCl}_{3}$ on $500 \mathrm{MHz} \mathrm{NMR}$ instrument 


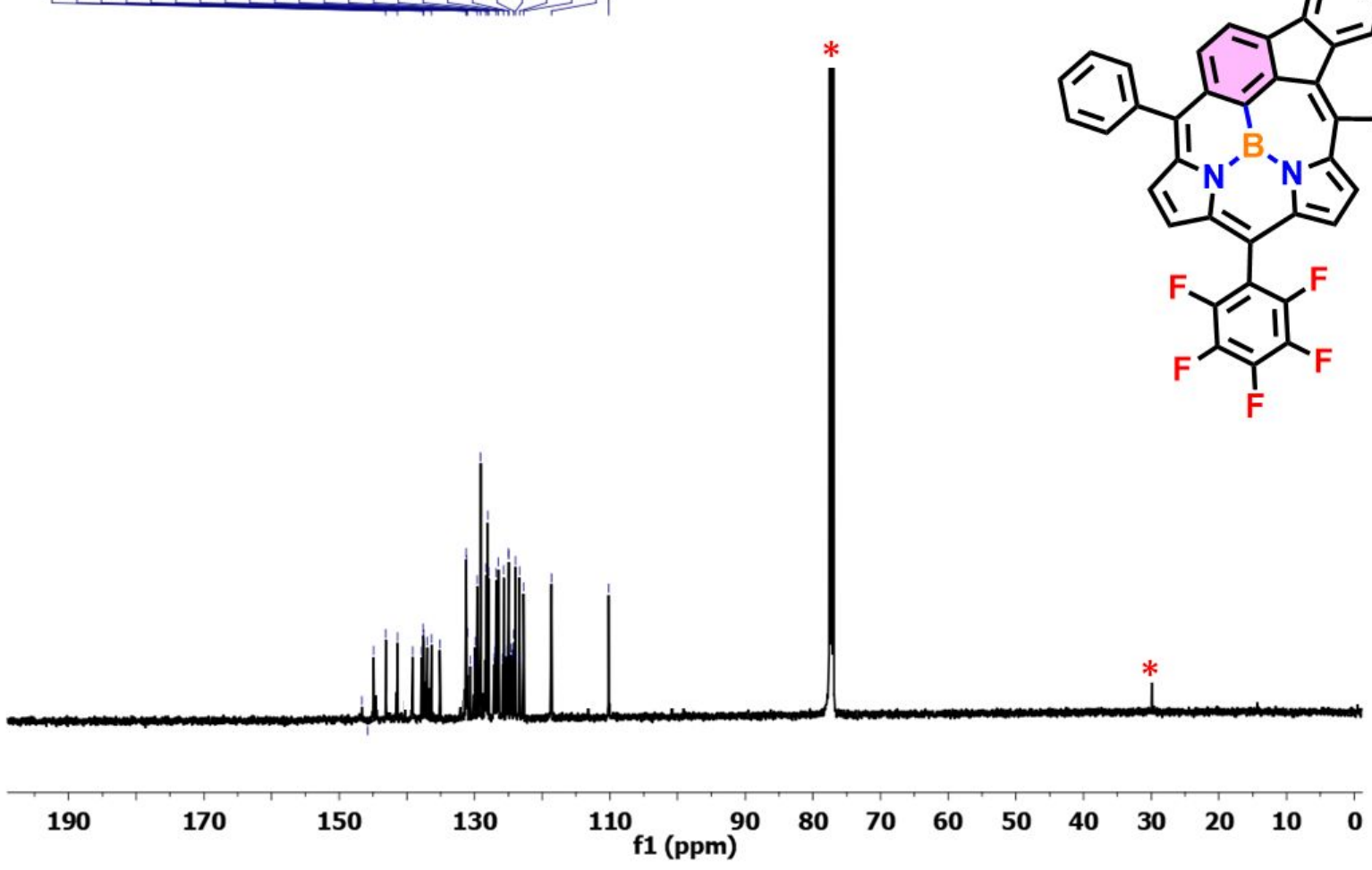

Figure S7. ${ }^{13} \mathrm{C}\left\{{ }^{1} \mathrm{H}\right\}$ NMR spectrum of the compound 10 recorded in $\mathrm{CDCl}_{3}$ on $500 \mathrm{MHz} \mathrm{NMR}$ instrument; Note: Peaks marked with asterisk $\left(^{*}\right)$ are due to residual solvents. 


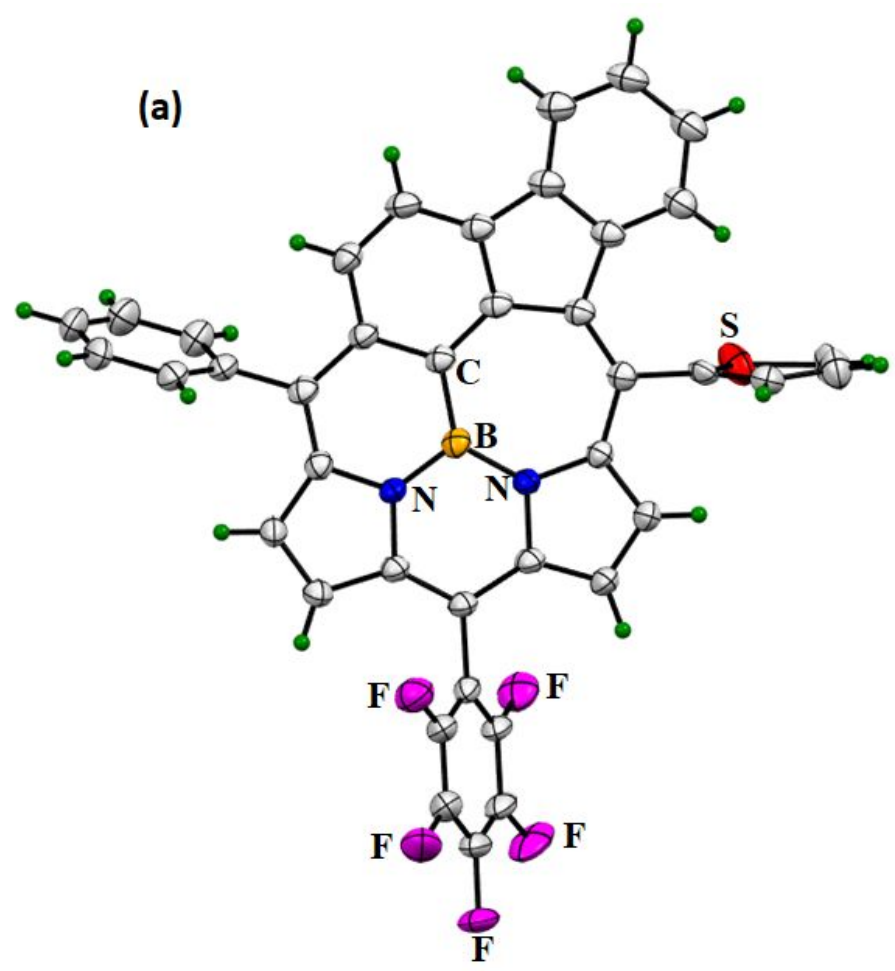

(b)

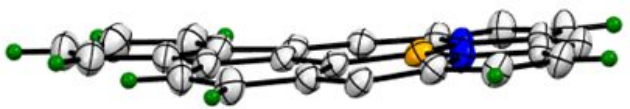

Figure S8. The ORTEP diagram of compound $\mathbf{1 0}$ at 50\% probability level. (a) top view; (b) side view; Note: The aryl groups on meso $\mathrm{sp}^{2}$ carbons in the side views were omitted for clarity. 
Table S1: Crystal data and structure refinement for Compound 10

\begin{tabular}{|c|c|}
\hline Identification code & FLU-B \\
\hline CCDC no & 2106226 \\
\hline Molecular Formula & $\mathrm{C}_{40} \mathrm{H}_{18} \mathrm{BF}_{5} \mathrm{~N}_{2} \mathrm{~S}$ \\
\hline Temperature/K & $293(2)$ \\
\hline Crystal system & monoclinic \\
\hline Space group & $\mathrm{P} 2{ }_{1} / \mathrm{c}$ \\
\hline $\mathrm{a} / \AA$ & $11.8242(7)$ \\
\hline $\mathrm{b} / \AA$ & $12.9897(6)$ \\
\hline $\mathrm{c} / \AA$ & $19.3054(8)$ \\
\hline$\alpha /^{\circ}$ & 90 \\
\hline$\beta /{ }^{\circ}$ & $94.918(5)$ \\
\hline$\gamma /{ }^{\circ}$ & 90 \\
\hline Volume $/ \AA^{3}$ & 2954.3(3) \\
\hline Z & 4 \\
\hline$\rho_{\text {calc }} \mathrm{g} / \mathrm{cm}^{3}$ & 1.504 \\
\hline$\mu / \mathrm{mm}^{-1}$ & 0.189 \\
\hline $\mathrm{F}(000)$ & 1360.0 \\
\hline Crystal size $/ \mathrm{mm}^{3}$ & $0.148 \times 0.110 \times 0.080$ \\
\hline Radiation & $\mathrm{M}_{\mathrm{o}} \mathrm{K}_{\alpha}(\lambda=0.71073)$ \\
\hline $2 \Theta$ range for data collection $/{ }^{\circ}$ & 4.668 to 50 \\
\hline Index ranges & $-12 \leq \mathrm{h} \leq 14,-11 \leq \mathrm{k} \leq 15,-22 \leq 1 \leq 15$ \\
\hline Reflections collected & 18056 \\
\hline Independent reflections & $5157\left[\mathrm{R}_{\text {int }}=0.1316, \mathrm{R}_{\text {sigma }}=0.0766\right]$ \\
\hline Data/restraints/parameters & $5157 / 598 / 544$ \\
\hline Goodness-of-fit on $\mathrm{F}^{2}$ & 1.098 \\
\hline Final R indexes $[\mathrm{I}>=2 \sigma(\mathrm{I})]$ & $\mathrm{R}_{1}=0.0563, \mathrm{wR}_{2}=0.1387$ \\
\hline Final R indexes [all data] & $\mathrm{R}_{1}=0.0878, \mathrm{wR}_{2}=0.1528$ \\
\hline Largest diff. peak/hole / e $\AA^{-3}$ & $0.45 /-0.44$ \\
\hline
\end{tabular}


Table S2. Bond Lengths for 10.

\begin{tabular}{|llrllr|}
\hline Atom Atom & Length/A & Atom Atom & Length/A \\
\hline N1 & B1 & $1.437(4)$ & C24 & C25 & $1.488(4)$ \\
N1 & C15 & $1.401(3)$ & C44 & C43 & $1.351(4)$ \\
N1 & C18 & $1.412(3)$ & C44 & S2 & $1.725(4)$ \\
N2 & B1 & $1.418(4)$ & C43 & C41 & $1.334(4)$ \\
N2 & C20 & $1.398(3)$ & C41 & C42 & $1.346(4)$ \\
N2 & C23 & $1.396(3)$ & C42 & S2 & $1.716(3)$ \\
B1 & C2 & $1.522(4)$ & C31 & C32 & $1.357(5)$ \\
C2 & C3 & $1.433(4)$ & C31 & S1 & $1.707(5)$ \\
C2 & C1 & $1.402(4)$ & C32 & C33 & $1.356(5)$ \\
C3 & C4 & $1.413(4)$ & C33 & C34 & $1.348(5)$ \\
C3 & C24 & $1.461(4)$ & C34 & S1 & $1.718(5)$ \\
C4 & C5 & $1.379(4)$ & F5 & C40 & $1.309(7)$ \\
C1 & C6 & $1.405(3)$ & F4 & C39 & $1.324(5)$ \\
C1 & C13 & $1.492(4)$ & F3 & C38 & $1.343(4)$ \\
C6 & C5 & $1.390(4)$ & F2 & C37 & $1.341(5)$ \\
C6 & C7 & $1.459(4)$ & F1 & C36 & $1.318(7)$ \\
C8 & C9 & $1.378(4)$ & C35 & C40 & 1.3900 \\
C8 & C7 & $1.387(4)$ & C35 & C36 & 1.3900 \\
C9 & C10 & $1.384(4)$ & C40 & C39 & 1.3900 \\
C10 & C11 & $1.385(4)$ & C39 & C38 & 1.3900 \\
C7 & C12 & $1.415(4)$ & C38 & C37 & 1.3900 \\
C12 & C11 & $1.409(4)$ & C37 & C36 & 1.3900 \\
C12 & C13 & $1.486(4)$ & F1A & C26A & $1.332(17)$ \\
C13 & C14 & $1.377(4)$ & F2A & C27A & $1.379(13)$ \\
C14 & C15 & $1.453(3)$ & F3A & C28A & $1.364(10)$ \\
C14 & C44 & $1.472(6)$ & F4A & C29A & $1.322(14)$ \\
C14 & C31 & $1.607(17)$ & F5A & C30A & $1.308(18)$ \\
C15 & C16 & $1.390(4)$ & C25AC26A & 1.3900 \\
C16 & C17 & $1.391(4)$ & C25A C30A & 1.3900 \\
C17 & C11 & $1.779(5)$ & C26A C27A & 1.3900 \\
C17 & C18 & $1.386(4)$ & C27A C28A & 1.3900 \\
C18 & C19 & $1.433(3)$ & C28A C29A & 1.3900 \\
C19 & C20 & $1.372(4)$ & C29A C30A & 1.3900 \\
C19 & C35 & $1.520(4)$ & C25 & C26 & $1.392(4)$ \\
C19 & C25A & $1.467(7)$ & C25 & C30 & $1.397(4)$ \\
C20 & C21 & $1.441(4)$ & C26 & C27 & $1.394(4)$ \\
C21 & C22 & $1.354(4)$ & C27 & C28 & $1.375(5)$ \\
C23 & $1.373(4)$ & C29 & C30 & $1.380(4)$ \\
\hline
\end{tabular}


Table S3. Bond Angles for 10.

\begin{tabular}{|c|c|c|c|c|c|c|c|}
\hline \multicolumn{3}{|c|}{ Atom Atom Atom } & \multirow{2}{*}{$\frac{\text { Angle }^{\circ}}{131.0(2)}$} & \multicolumn{3}{|c|}{ Atom Atom Atom } & \multirow{2}{*}{$\frac{\text { Angle }^{\circ}}{133.6(2)}$} \\
\hline C15 & N1 & B1 & & $\mathrm{C} 24$ & $\mathrm{C} 23$ & $\mathrm{C} 22$ & \\
\hline C15 & N1 & $\mathrm{C} 18$ & $108.2(2)$ & $\mathrm{C} 3$ & $\mathrm{C} 24$ & $\mathrm{C} 25$ & $121.1(2)$ \\
\hline C18 & N1 & B1 & $120.8(2)$ & $\mathrm{C} 23$ & $\mathrm{C} 24$ & $\mathrm{C} 3$ & $120.3(2)$ \\
\hline $\mathrm{C} 20$ & $\mathrm{~N} 2$ & B1 & $125.5(2)$ & $\mathrm{C} 23$ & $\mathrm{C} 24$ & $\mathrm{C} 25$ & $118.4(2)$ \\
\hline C23 & N2 & B1 & $124.4(2)$ & C14 & C44 & $\mathrm{S} 2$ & $120.8(3)$ \\
\hline C23 & N2 & $\mathrm{C} 20$ & $109.9(2)$ & $\mathrm{C} 43$ & C44 & $\mathrm{C} 14$ & $137.4(4)$ \\
\hline N1 & B1 & $\mathrm{C} 2$ & $129.1(2)$ & $\mathrm{C} 43$ & C44 & $\mathrm{S} 2$ & $101.6(4)$ \\
\hline $\mathrm{N} 2$ & B1 & N1 & $114.6(2)$ & $\mathrm{C} 41$ & $\mathrm{C} 43$ & $\mathrm{C} 44$ & $125.7(6)$ \\
\hline $\mathrm{N} 2$ & B1 & $\mathrm{C} 2$ & $116.3(2)$ & $\mathrm{C} 43$ & $\mathrm{C} 41$ & $\mathrm{C} 42$ & $105.5(4)$ \\
\hline C3 & $\mathrm{C} 2$ & B1 & $117.8(2)$ & $\mathrm{C} 41$ & $\mathrm{C} 42$ & $\mathrm{~S} 2$ & $112.9(3)$ \\
\hline C1 & $\mathrm{C} 2$ & B1 & $123.4(2)$ & $\mathrm{C} 42$ & S2 & $\mathrm{C} 44$ & $93.3(2)$ \\
\hline C1 & $\mathrm{C} 2$ & $\mathrm{C} 3$ & $118.7(2)$ & C14 & $\mathrm{C} 31$ & S1 & $111.0(8)$ \\
\hline $\mathrm{C} 2$ & $\mathrm{C} 3$ & $\mathrm{C} 24$ & $120.7(2)$ & $\mathrm{C} 32$ & $\mathrm{C} 31$ & $\mathrm{C} 14$ & $123.0(9)$ \\
\hline C4 & $\mathrm{C} 3$ & $\mathrm{C} 2$ & $118.9(2)$ & $\mathrm{C} 32$ & $\mathrm{C} 31$ & $\mathrm{~S} 1$ & $126.0(13)$ \\
\hline C4 & $\mathrm{C} 3$ & $\mathrm{C} 24$ & $120.3(2)$ & $\mathrm{C} 33$ & $\mathrm{C} 32$ & $\mathrm{C} 31$ & $102.0(15)$ \\
\hline C5 & $\mathrm{C} 4$ & $\mathrm{C} 3$ & $121.5(3)$ & $\mathrm{C} 34$ & $\mathrm{C} 33$ & $\mathrm{C} 32$ & $113.8(13)$ \\
\hline C2 & $\mathrm{C} 1$ & C6 & $120.4(2)$ & C33 & C34 & S1 & $118.7(10)$ \\
\hline $\mathrm{C} 2$ & $\mathrm{C} 1$ & $\mathrm{C} 13$ & $130.7(2)$ & $\mathrm{C} 31$ & S1 & $\mathrm{C} 34$ & $79.2(8)$ \\
\hline C6 & $\mathrm{C} 1$ & $\mathrm{C} 13$ & $108.8(2)$ & $\mathrm{C} 40$ & $\mathrm{C} 35$ & $\mathrm{C} 19$ & $121.8(4)$ \\
\hline $\mathrm{C} 1$ & $\mathrm{C} 6$ & $\mathrm{C} 7$ & $108.9(2)$ & $\mathrm{C} 40$ & C35 & $\mathrm{C} 36$ & 120.0 \\
\hline C5 & $\mathrm{C} 6$ & $\mathrm{C} 1$ & $120.8(2)$ & $\mathrm{C} 36$ & $\mathrm{C} 35$ & $\mathrm{C} 19$ & $118.2(4)$ \\
\hline C5 & C6 & $\mathrm{C} 7$ & $130.2(2)$ & F5 & $\mathrm{C} 40$ & $\mathrm{C} 35$ & $120.4(4)$ \\
\hline C4 & $\mathrm{C} 5$ & $\mathrm{C} 6$ & $119.5(2)$ & F5 & $\mathrm{C} 40$ & C39 & $119.6(4)$ \\
\hline C9 & $\mathrm{C} 8$ & $\mathrm{C} 7$ & $119.1(3)$ & $\mathrm{C} 35$ & $\mathrm{C} 40$ & C39 & 120.0 \\
\hline C8 & $\mathrm{C} 9$ & $\mathrm{C} 10$ & $120.2(3)$ & F4 & C39 & $\mathrm{C} 40$ & $119.6(3)$ \\
\hline C9 & $\mathrm{C} 10$ & $\mathrm{C} 11$ & $121.8(3)$ & F4 & C39 & $\mathrm{C} 38$ & $120.4(3)$ \\
\hline C8 & $\mathrm{C} 7$ & $\mathrm{C} 6$ & $129.9(3)$ & $\mathrm{C} 40$ & C39 & $\mathrm{C} 38$ & 120.0 \\
\hline C8 & $\mathrm{C} 7$ & $\mathrm{C} 12$ & $121.7(3)$ & F3 & $\mathrm{C} 38$ & C39 & $119.7(3)$ \\
\hline C12 & $\mathrm{C} 7$ & C6 & $108.5(2)$ & F3 & $\mathrm{C} 38$ & C37 & $120.2(3)$ \\
\hline C7 & $\mathrm{C} 12$ & $\mathrm{C} 13$ & $108.8(2)$ & C39 & $\mathrm{C} 38$ & $\mathrm{C} 37$ & 120.0 \\
\hline C11 & $\mathrm{C} 12$ & $\mathrm{C} 7$ & $118.1(2)$ & $\mathrm{F} 2$ & C37 & $\mathrm{C} 38$ & $119.2(3)$ \\
\hline C11 & $\mathrm{C} 12$ & $\mathrm{C} 13$ & $133.1(3)$ & F2 & $\mathrm{C} 37$ & $\mathrm{C} 36$ & $120.8(3)$ \\
\hline C10 & $\mathrm{C} 11$ & $\mathrm{C} 12$ & $119.1(3)$ & $\mathrm{C} 38$ & $\mathrm{C} 37$ & $\mathrm{C} 36$ & 120.0 \\
\hline C12 & $\mathrm{C} 13$ & $\mathrm{C} 1$ & $105.0(2)$ & F1 & $\mathrm{C} 36$ & $\mathrm{C} 35$ & $123.0(5)$ \\
\hline C14 & $\mathrm{C} 13$ & $\mathrm{C} 1$ & $128.8(2)$ & F1 & C36 & C37 & $117.0(5)$ \\
\hline C14 & $\mathrm{C} 13$ & $\mathrm{C} 12$ & $126.1(2)$ & C37 & $\mathrm{C} 36$ & $\mathrm{C} 35$ & 120.0 \\
\hline C13 & $\mathrm{C} 14$ & $\mathrm{C} 15$ & $129.9(2)$ & $\mathrm{C} 26 \mathrm{~A}$ & $\mathrm{C} 25 \mathrm{~A}$ & $\mathrm{AC} 19$ & $115.1(9)$ \\
\hline
\end{tabular}


Table S3. Bond Angles for 10.

\begin{tabular}{|c|c|c|c|c|c|}
\hline \multicolumn{3}{|c|}{ Atom Atom Atom } & \multirow{2}{*}{$\frac{\text { Angle }^{\circ}}{121.0(3)}$} & \multirow{2}{*}{$\frac{\text { Atom Atom Atom }}{\text { C26A C25A C30A }}$} & \multirow{2}{*}{$\frac{\text { Angle }^{\circ}}{120.0}$} \\
\hline C13 & $\mathrm{C} 14$ & $\mathrm{C} 44$ & & & \\
\hline C13 & $\mathrm{C} 14$ & $\mathrm{C} 31$ & $112.4(7)$ & C30A C 25 A C 19 & $124.8(9)$ \\
\hline C15 & C14 & $\mathrm{C} 44$ & $109.2(3)$ & F1A C26AC25A & $127.0(11)$ \\
\hline C15 & C14 & C31 & $116.9(7)$ & F1A C26AC27A & $112.9(11)$ \\
\hline N1 & $\mathrm{C} 15$ & $\mathrm{C} 14$ & $126.6(2)$ & C27A C26A C25A & 120.0 \\
\hline C16 & $\mathrm{C} 15$ & N1 & $107.0(2)$ & F2A C27AC26A & $123.9(8)$ \\
\hline C16 & $\mathrm{C} 15$ & $\mathrm{C} 14$ & $126.4(2)$ & F2A C27AC28A & $116.1(8)$ \\
\hline C15 & $\mathrm{C} 16$ & $\mathrm{C} 17$ & $109.3(2)$ & C26A C27A C28A & 120.0 \\
\hline C16 & $\mathrm{C} 17$ & $\mathrm{Cl1}$ & $123.5(3)$ & F3A C28AC27A & $122.3(8)$ \\
\hline C18 & $\mathrm{C} 17$ & $\mathrm{C} 16$ & $108.0(2)$ & F3A C28AC29A & $117.6(8)$ \\
\hline C18 & $\mathrm{C} 17$ & $\mathrm{Cl1}$ & $125.9(3)$ & C29A C28A C27A & 120.0 \\
\hline N1 & $\mathrm{C} 18$ & $\mathrm{C} 19$ & $120.6(2)$ & F4A C29AC28A & $121.3(8)$ \\
\hline C17 & $\mathrm{C} 18$ & N1 & $107.6(2)$ & F4A C29AC30A & $118.6(8)$ \\
\hline C17 & $\mathrm{C} 18$ & C19 & $131.8(2)$ & C28A C29A C30A & 120.0 \\
\hline C18 & C19 & C35 & $119.5(4)$ & F5A C30AC25A & $117.3(13)$ \\
\hline C18 & C19 & $\mathrm{C} 25 \mathrm{~A}$ & $119.4(9)$ & F5A C30AC29A & $122.7(13)$ \\
\hline $\mathrm{C} 20$ & C19 & $\mathrm{C} 18$ & $119.8(2)$ & C29A C30A C25A & 120.0 \\
\hline $\mathrm{C} 20$ & C19 & C35 & $120.7(4)$ & $\mathrm{C} 26 \quad \mathrm{C} 25 \quad \mathrm{C} 24$ & $121.7(3)$ \\
\hline $\mathrm{C} 20$ & $\mathrm{C} 19$ & $\mathrm{C} 25 \mathrm{~A}$ & $120.5(10)$ & $\mathrm{C} 26 \quad \mathrm{C} 25 \quad \mathrm{C} 30$ & $118.3(3)$ \\
\hline $\mathrm{N} 2$ & $\mathrm{C} 20$ & $\mathrm{C} 21$ & $106.3(2)$ & $\mathrm{C} 30 \quad \mathrm{C} 25 \quad \mathrm{C} 24$ & $120.0(3)$ \\
\hline C19 & $\mathrm{C} 20$ & $\mathrm{~N} 2$ & $118.6(2)$ & $\mathrm{C} 25 \quad \mathrm{C} 26 \quad \mathrm{C} 27$ & $119.9(3)$ \\
\hline C19 & $\mathrm{C} 20$ & $\mathrm{C} 21$ & $135.1(2)$ & $\mathrm{C} 28 \quad \mathrm{C} 27 \quad \mathrm{C} 26$ & $120.7(3)$ \\
\hline $\mathrm{C} 22$ & $\mathrm{C} 21$ & $\mathrm{C} 20$ & $108.4(2)$ & $\begin{array}{lll}\mathrm{C} 27 & \mathrm{C} 28 & \mathrm{C} 29\end{array}$ & $120.0(3)$ \\
\hline C21 & $\mathrm{C} 22$ & $\mathrm{C} 23$ & $109.5(2)$ & C28 C29 C30 & $119.7(3)$ \\
\hline $\mathrm{N} 2$ & $\mathrm{C} 23$ & $\mathrm{C} 22$ & $105.8(2)$ & C29 C30 C25 & $121.4(3)$ \\
\hline $\mathrm{C} 24$ & $\mathrm{C} 23$ & $\mathrm{~N} 2$ & $120.4(2)$ & & \\
\hline
\end{tabular}

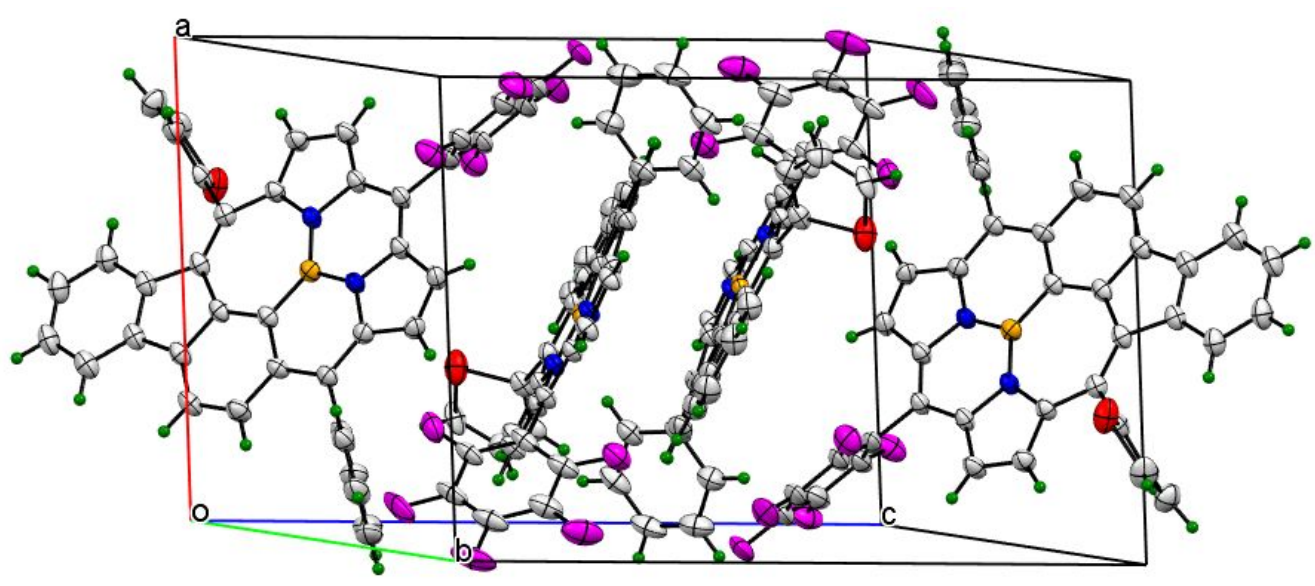


Figure S9. Crystal packing diagram of compound $\mathbf{1 0 .}$

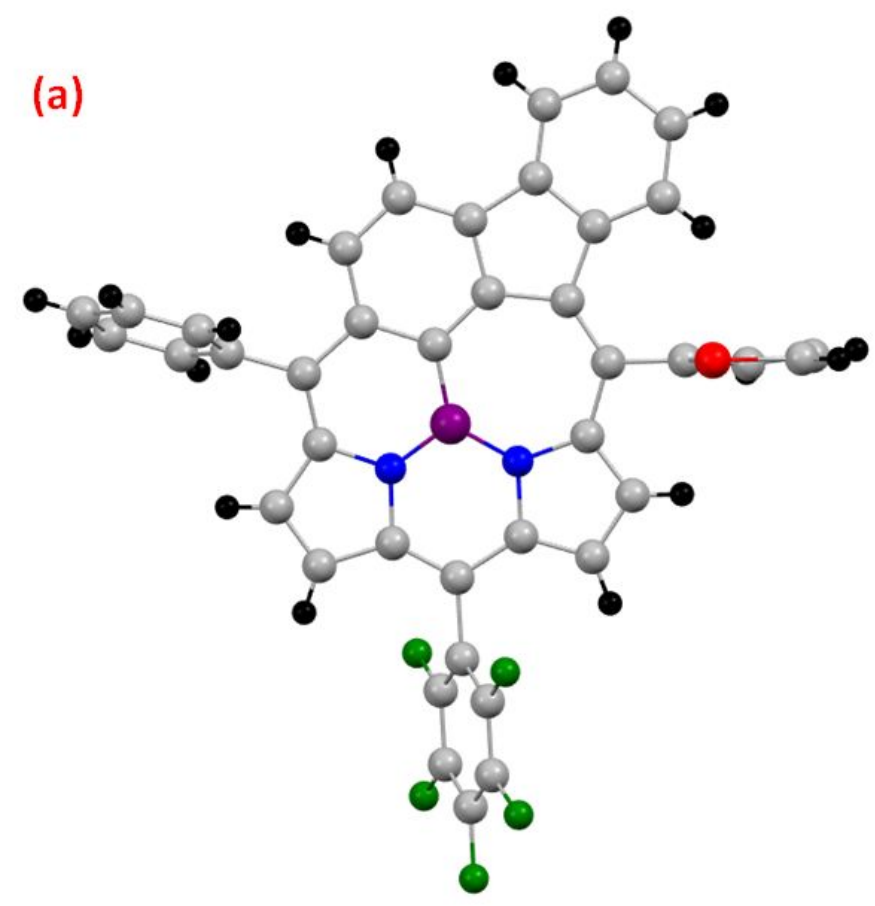

(b)

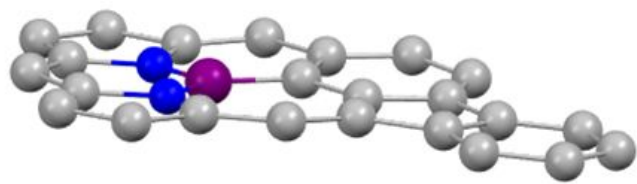

Figure S10. Optimized (B3LYP/6-31G $(\mathrm{d}, \mathrm{p})$ geometries of the compounds $\mathbf{1 0}$ (a) top view and (b) side view; Note- Hydrogen atoms in the top views and side views were omitted for clarity 
(A)

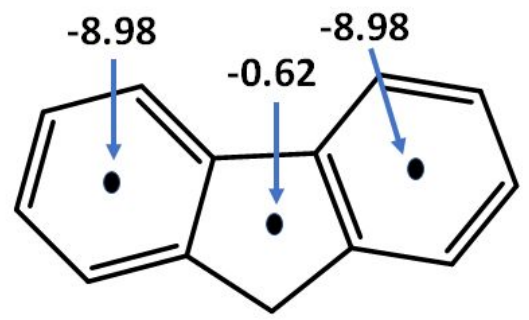

(B)

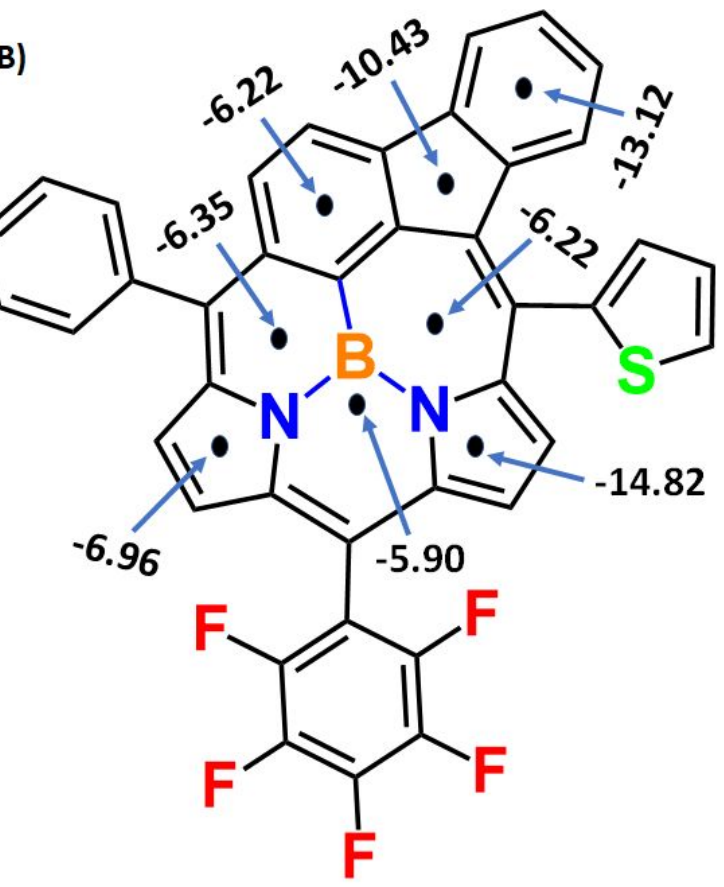

Figure S11. NICS values of (A) fluorene and (B) compound 10 on the optimized structures (B3LYP/6-31G(d,p)( black dots represent the Bq atoms).

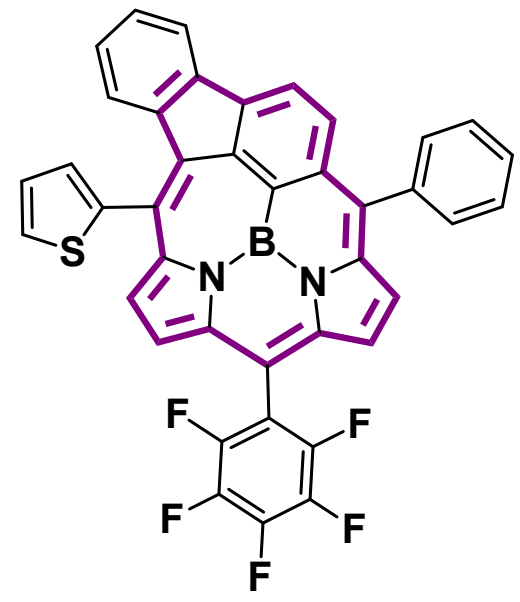

10-a $(18 \pi)$

HOMA $=1.15$

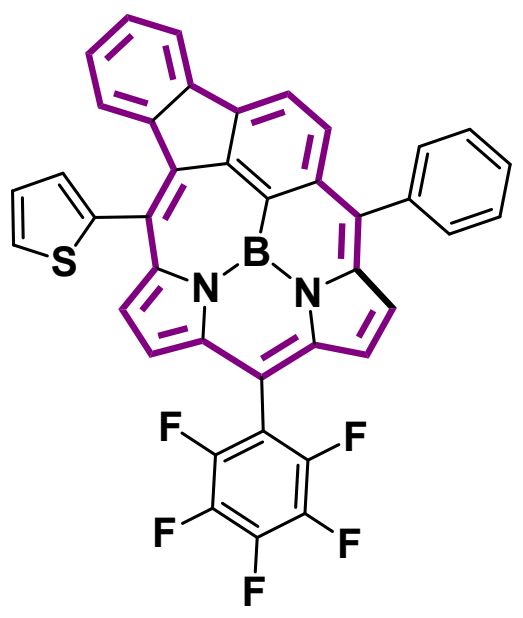

10-b $(22 \pi)$

1.12

Figure S12. HOMA indices for various pathways of compound 10. The $\alpha$ and $\mathrm{R}_{\mathrm{opt}}(\AA)$ values were taken as, 257.70, 93.52 and 1.388, 1.334 respectively for C-C and C-N. 
(A)

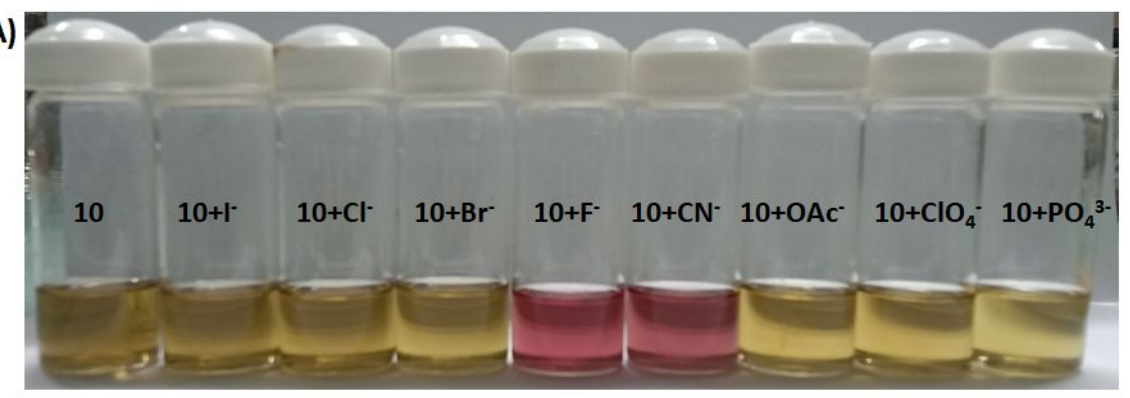

(B)

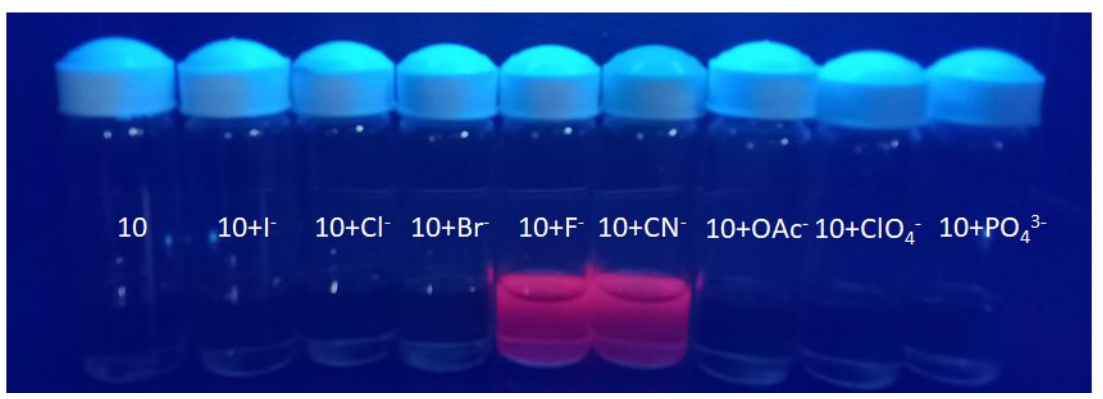

Figure S13. Colour change induced upon addition of different anions in excess (as the $\mathrm{Bu}_{4} \mathrm{~N}^{+}$ salt) to the $\mathrm{CHCl}_{3}$ solution of $\mathbf{1 0}$ under (A) day light (B) long range UV light.
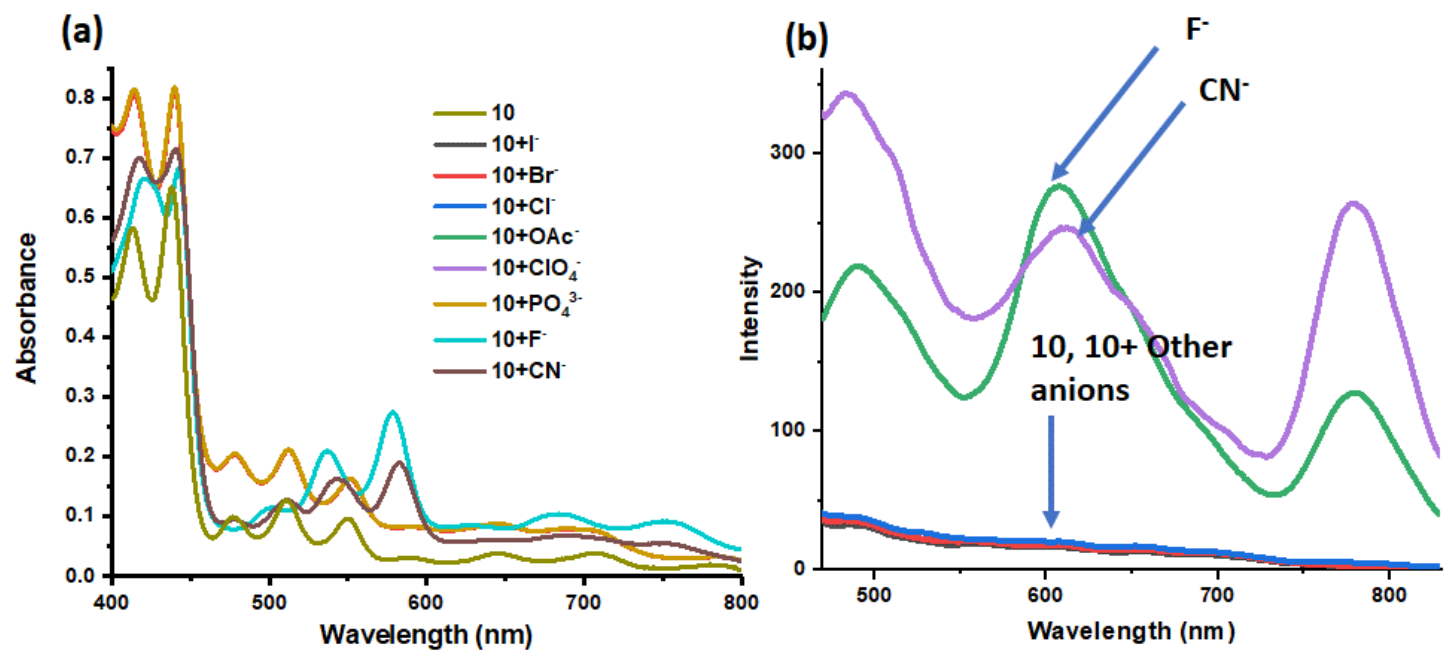

Figure S14: The (a) absorption spectra (b) fluorescence spectra of $10\left(1 \times 10^{-5} \mathrm{M}\right)$ in presence of various anions (excess of equivalents). 


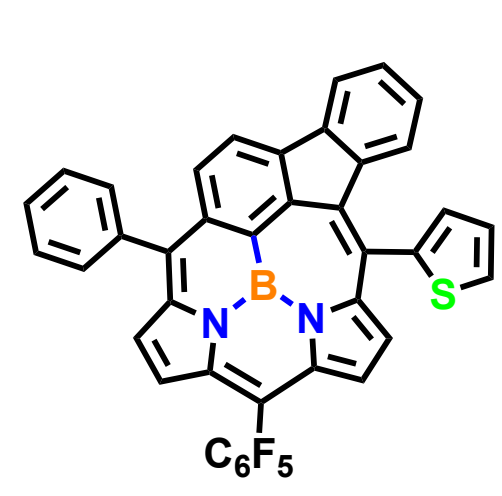

10

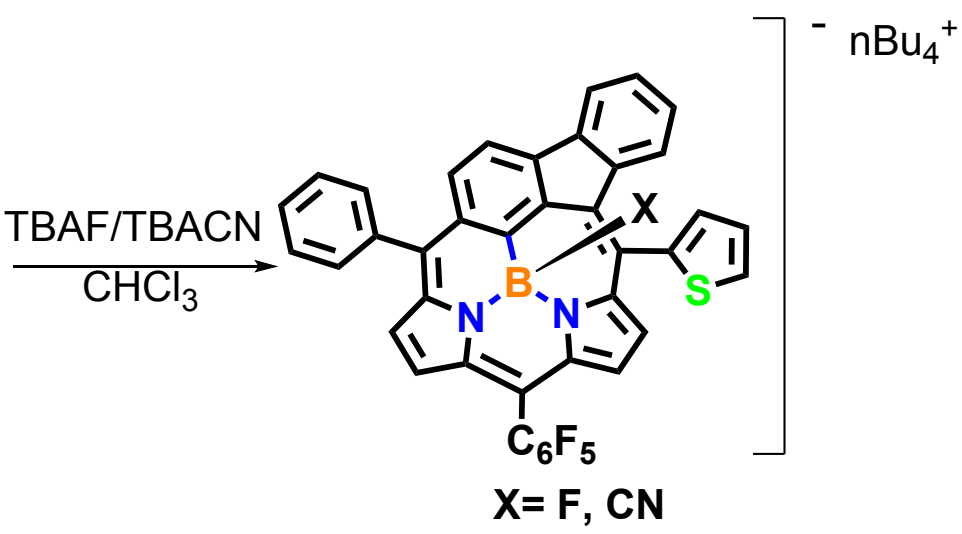

10.X

Scheme S1. Plausible mechanism for the sensing of $\mathrm{F}^{-} / \mathrm{CN}^{-}$ions by B(III)complex 10.

Table S4. Absorption and Emission data of 10, 10.F- $10 . \mathrm{CN}^{-}$

\begin{tabular}{|c|c|c|c|c|c|c|c|c|c|c|}
\hline \multirow[t]{2}{*}{ Compound } & \multicolumn{9}{|c|}{$\begin{array}{c}\text { Absorption data } \\
\lambda_{\text {abs }}(\mathbf{n m}) \\
\end{array}$} & \multirow[t]{2}{*}{$\begin{array}{c}\text { Emission data } \\
\lambda_{\text {ems }}(\mathbf{n m})\end{array}$} \\
\hline & & ret ba & & & & -banc & & & & \\
\hline 10 & 414 & 440 & 476 & 510 & 549 & 589 & 644 & 707 & 785 & - \\
\hline $10 . F^{-}$ & 417 & 442 & 505 & 537 & 579 & 627 & 683 & 749 & - & $482,621,780$ \\
\hline 10. $\mathrm{CN}^{-}$ & 429 & 446 & 503 & 541 & 583 & 627 & 685 & 754 & - & $483,609,778$ \\
\hline
\end{tabular}


(a)

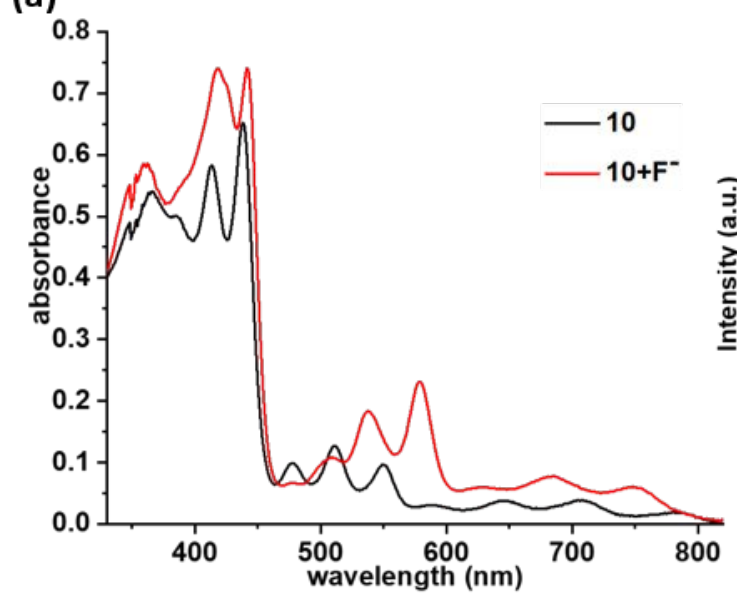

(c)

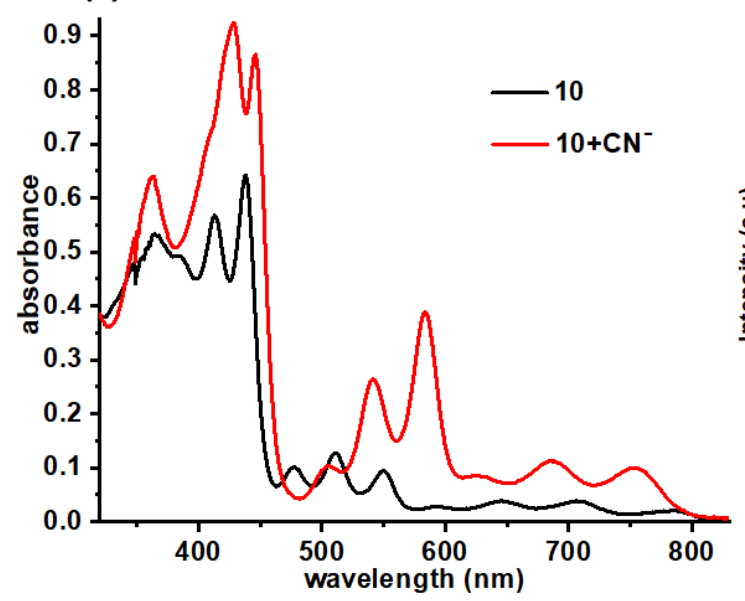

(b)

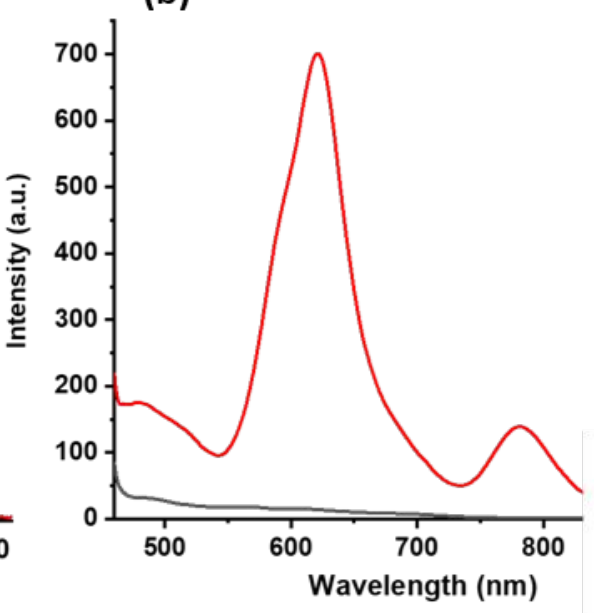

(d)

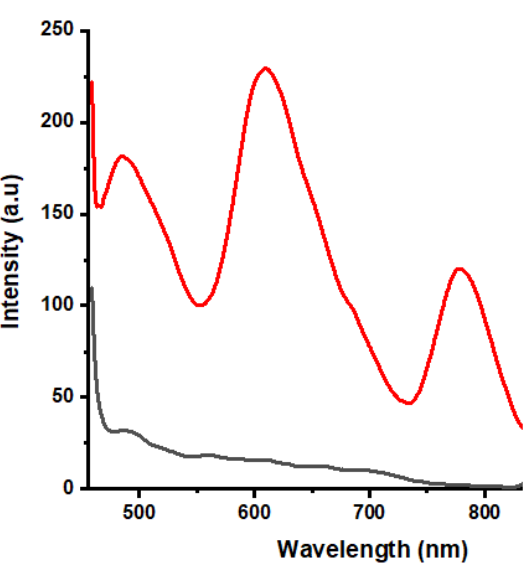

Figure S15: (a) The absorption spectra (b) emission spectra of $10\left(1 \times 10^{-5}\right)$ in the presence of excess equivalents of $\mathrm{F}^{-}$(TBAF) in $\mathrm{CHCl}_{3}$. (a) The absorption spectra (b) emission spectra of 10 in the presence of excess equivalents of $\mathrm{CN}^{-}(\mathrm{TBACN})$ in $\mathrm{CHCl}_{3}$ 

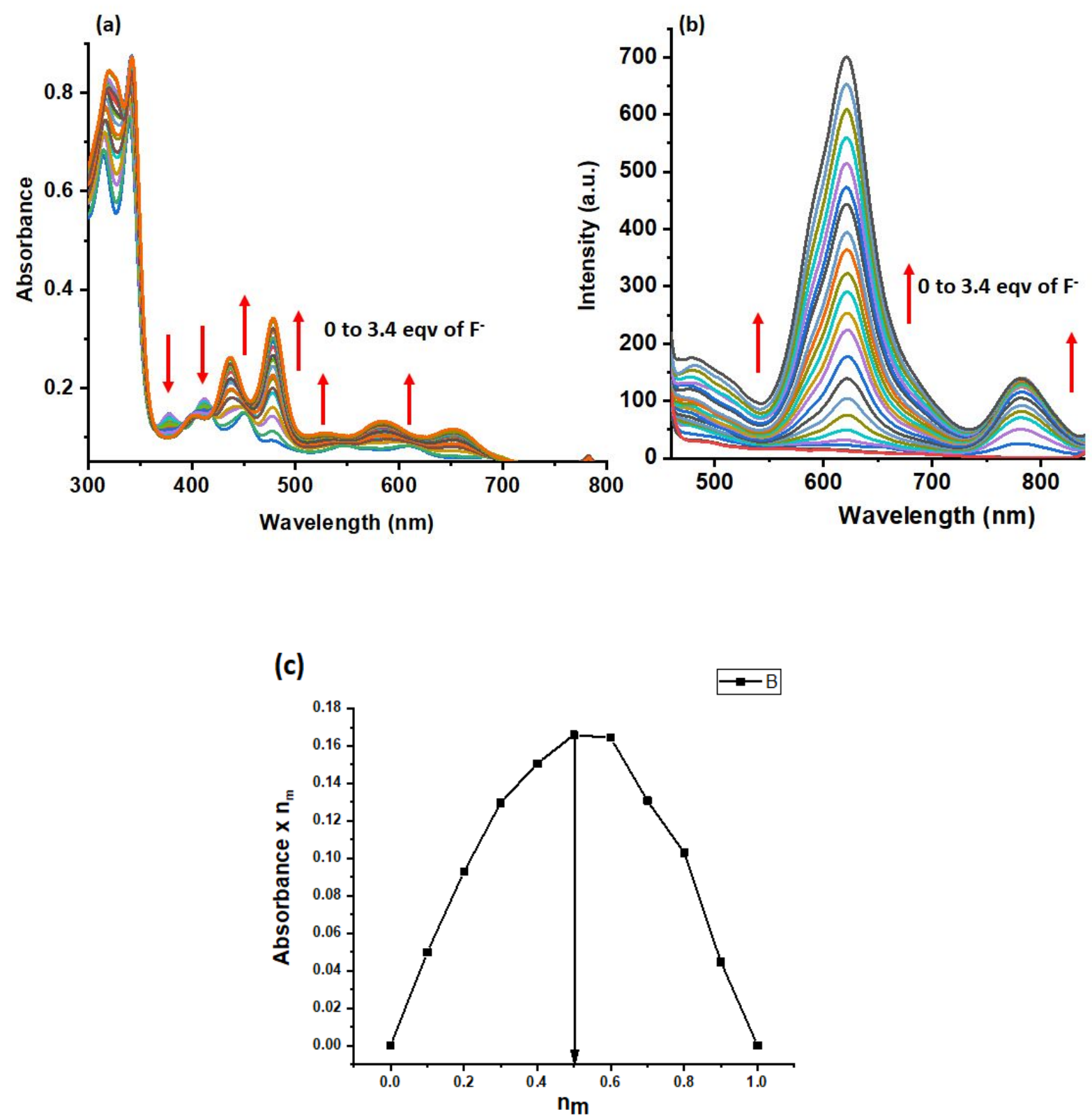

Figure S16: (a) Change in the absorption spectra of compound $10\left(1 \times 10^{-5} \mathrm{M}\right)$ upon titration with $\mathrm{F}-(\mathrm{TBAF})(\mathrm{b})$ Enhancement of fluorescence intensity of compound $10\left(1 \mathrm{X} 10^{-5} \mathrm{M}\right)$ during the titration with $\mathrm{F}^{-}$(TBAF) (excitation wavelength used was $420 \mathrm{~nm}$ ) (c) Job's plot of $\mathrm{nm}$ vs Absorbance ${ }^{*} n_{m}$, where $n_{m}$ is mole fraction of the fluoride ion added of compound $\mathbf{1 0}$. 

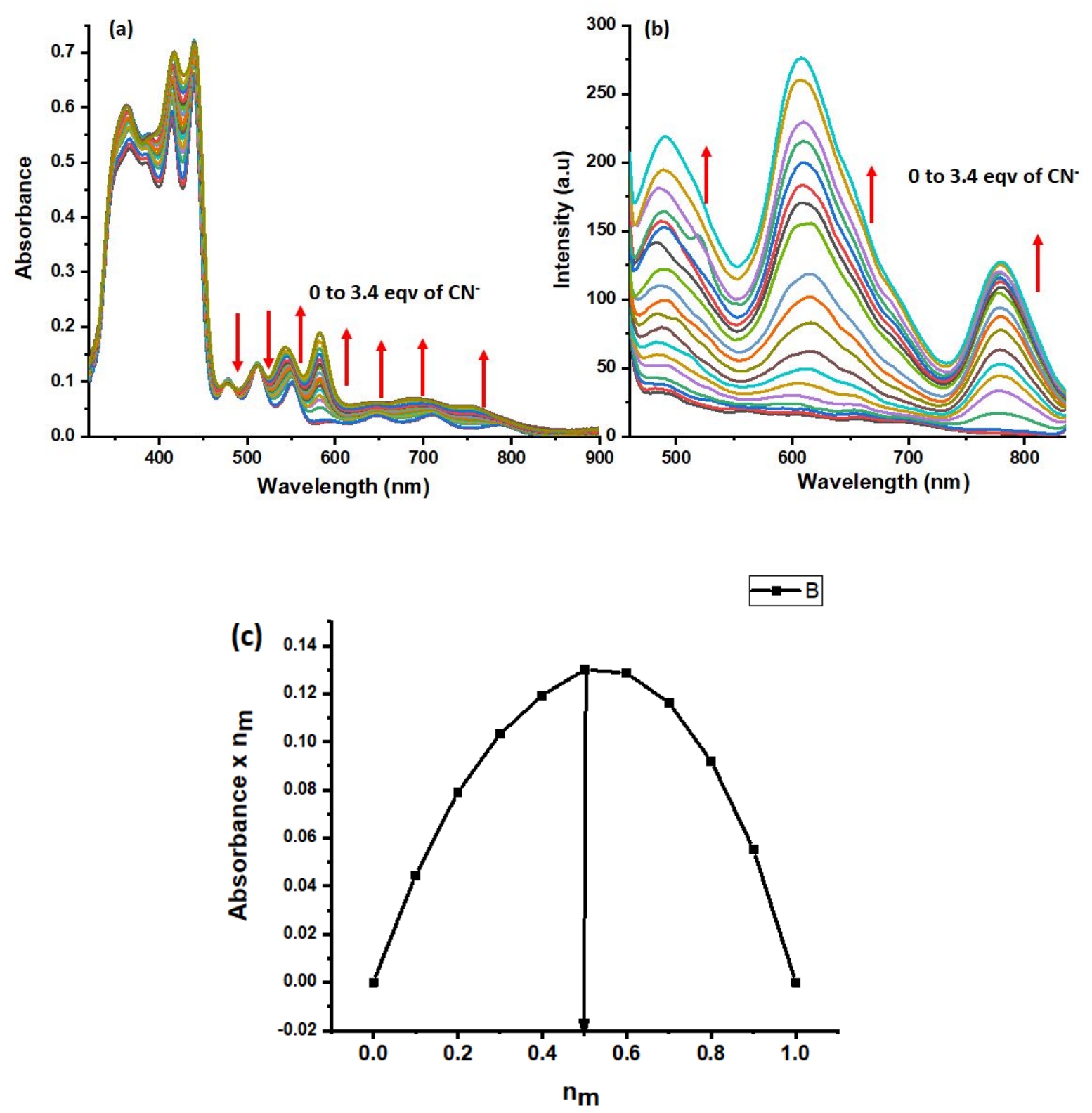

Figure S17: (a) Change in the absorption spectra of compound $10\left(1 \times 10^{-5} \mathrm{M}\right)$ upon titration with $\mathrm{CN}^{-}(\mathrm{TBACN})$ (b) Enhancement of fluorescence intensity of compound $10\left(1 \times 10^{-5} \mathrm{M}\right)$ during the titration with $\mathrm{F}^{-}$(TBACN) (excitation wavelength used was $420 \mathrm{~nm}$ ) (c) Job's plot of nm vs Absorbance $* \mathrm{n}_{\mathrm{m}}$, where $\mathrm{n}_{\mathrm{m}}$ is mole fraction of the fluoride ion added of compound $\mathbf{1 0}$. 

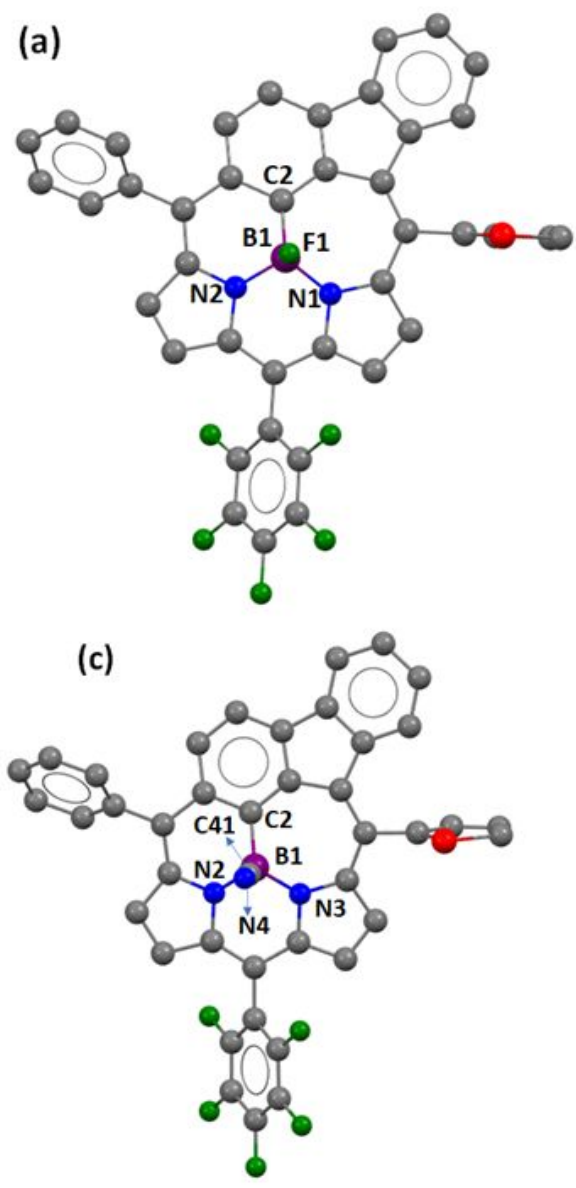

(b)

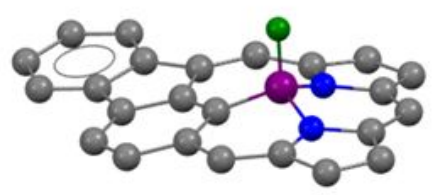

(d)

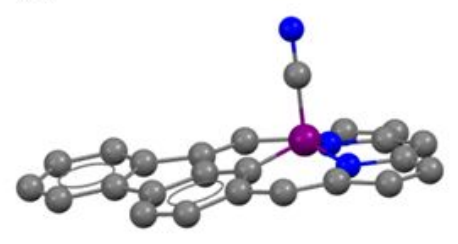

Figure S18. Optimized (B3LYP/6-31G(d,p) geometries of compounds 10.F- (a) top view and (b) side view and 10.CN- (c) top view (d) side view; Note- Hydrogen atoms in the top views and side views were omitted for clarity. 


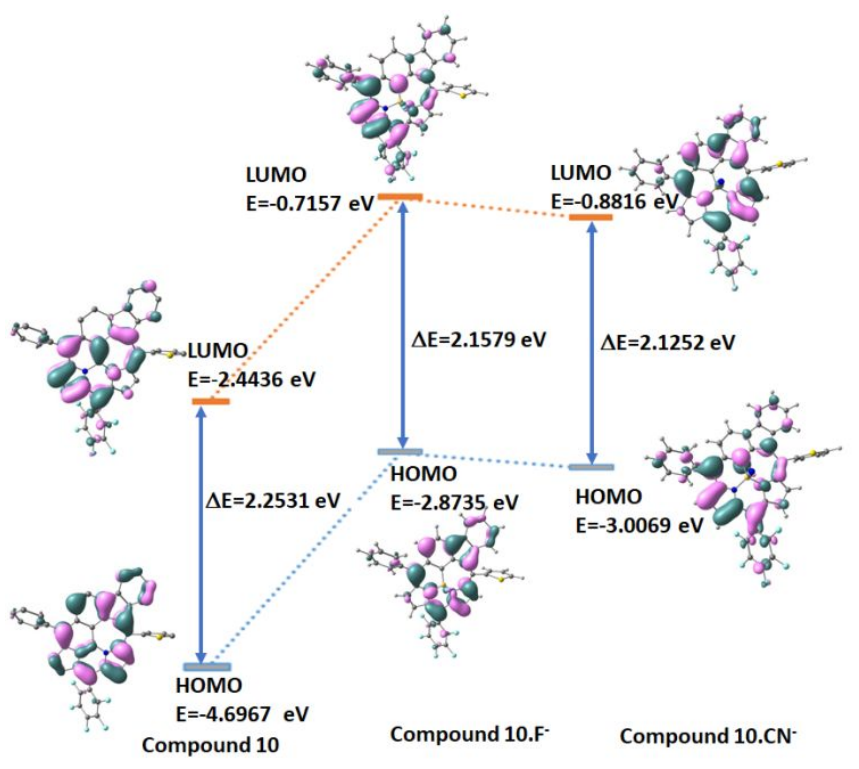

Figure S19. Energy-level diagram (selected FMOs) of compound 10, 10.F- and 10.CNcalculated by the B3LYP/6-31g $(\mathrm{d}, \mathrm{p})$ method.

(a)

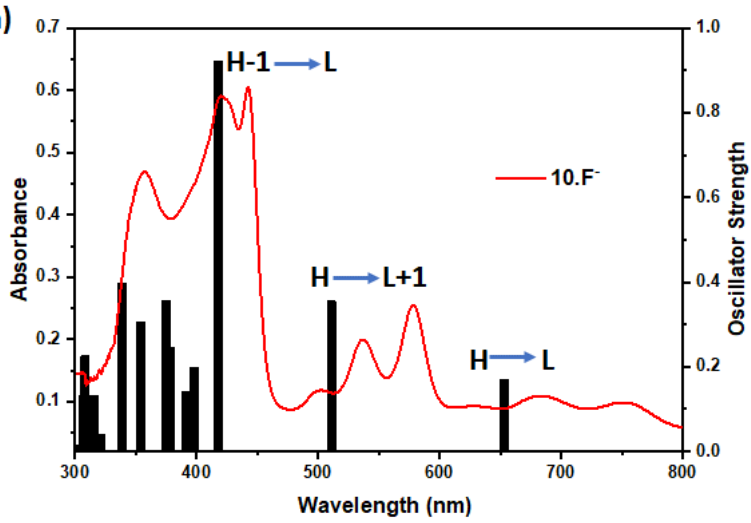

(b)

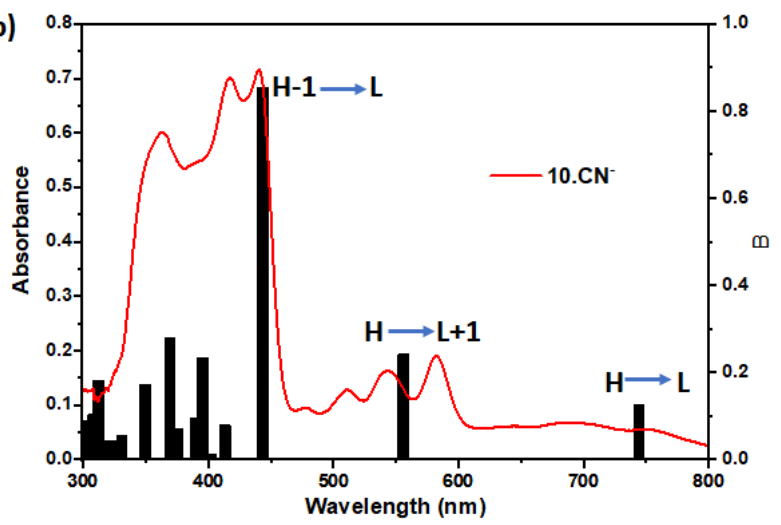

Figure S20. Calculated excitations (black vertical lines) and experimental UV/vis absorption spectra (red line) for compound (a) $\mathbf{1 0 . F ^ { - }}$ and (b) $\mathbf{1 0 . C N ^ { - }}$.

It can be seen from the above TD-DFT plots that the TD-DFT studies were well in accordance with the experimentally observed absorption spectrum of macrocycle $\mathbf{1 0 . F ^ { - }}$ and $\mathbf{1 0 . C N}$. 
Table S5. $\mathrm{S}_{0}$ optimized geometry of the compound 10 at B3LYP/6-31g (d,p) level of theory \# Sum of imaginary frequencies $=0$ \# Total Energy $($ hartree $)=-2566.935329$

\begin{tabular}{|c|c|c|c|c|c|c|c|}
\hline Atom & $\mathbf{X}$ & $Y$ & $\mathbf{Z}$ & Atom & $\mathbf{X}$ & $\mathbf{Y}$ & $\mathbf{Z}$ \\
\hline $\mathrm{N}$ & -0.07191 & -0.85646 & -0.09862 & $\mathrm{H}$ & -2.46991 & 4.288391 & 0.243059 \\
\hline $\mathrm{N}$ & -0.89257 & 1.398541 & -0.01957 & $\mathrm{C}$ & -0.82531 & 2.784232 & 0.069446 \\
\hline B & 0.245165 & 0.544324 & -0.05107 & $\mathrm{C}$ & 0.395819 & 3.430094 & 0.100265 \\
\hline $\mathrm{C}$ & 1.605885 & 1.225181 & -0.03412 & $\mathrm{C}$ & 2.735294 & -3.37589 & -0.20398 \\
\hline $\mathrm{C}$ & 1.632584 & 2.668016 & 0.029596 & $\mathrm{C}$ & 2.957827 & -4.18102 & -1.28827 \\
\hline $\mathrm{C}$ & 2.887105 & 3.334059 & -0.01256 & $\mathrm{H}$ & 2.769784 & -3.85141 & -2.30367 \\
\hline $\mathrm{H}$ & 2.914122 & 4.416241 & -0.00386 & $\mathrm{C}$ & 3.486209 & -5.46333 & -0.94072 \\
\hline $\mathrm{C}$ & 2.819415 & 0.524628 & -0.10608 & $\mathrm{H}$ & 3.737914 & -6.23026 & -1.66472 \\
\hline $\mathrm{C}$ & 4.044871 & 1.224995 & -0.11879 & $\mathrm{C}$ & 3.642136 & -5.62227 & 0.409302 \\
\hline $\mathrm{C}$ & 4.071537 & 2.624492 & -0.08078 & $\mathrm{H}$ & 4.012533 & -6.48632 & 0.944691 \\
\hline $\mathrm{H}$ & 5.0208 & 3.152339 & -0.11439 & S & 3.139697 & -4.20627 & 1.279787 \\
\hline $\mathrm{C}$ & 6.504655 & 0.500192 & -0.24432 & F & -3.49898 & -2.25266 & 1.862548 \\
\hline $\mathrm{H}$ & 6.891608 & 1.514881 & -0.21031 & F & -6.03711 & -3.15441 & 1.854946 \\
\hline $\mathrm{C}$ & 7.364602 & -0.58969 & -0.33704 & F & -7.82025 & -2.23096 & 0.004375 \\
\hline $\mathrm{H}$ & 8.439243 & -0.43588 & -0.37357 & F & -7.0236 & -0.40168 & -1.86017 \\
\hline $\mathrm{C}$ & 6.841048 & -1.89125 & -0.38938 & F & -4.48015 & 0.482908 & -1.89459 \\
\hline $\mathrm{H}$ & 7.516841 & -2.73784 & -0.46916 & $\mathrm{C}$ & -3.88819 & -0.85976 & -0.02311 \\
\hline $\mathrm{C}$ & 5.126679 & 0.271425 & -0.1984 & $\mathrm{C}$ & -4.33616 & -1.79312 & 0.921592 \\
\hline $\mathrm{C}$ & 4.576846 & -1.04154 & -0.23963 & $\mathrm{C}$ & -5.64575 & -2.26549 & 0.934186 \\
\hline $\mathrm{C}$ & 5.467354 & -2.12722 & -0.34396 & $\mathrm{C}$ & -6.55889 & -1.79163 & -0.005 \\
\hline $\mathrm{H}$ & 5.121164 & -3.14752 & -0.40437 & $\mathrm{C}$ & -6.15136 & -0.85468 & -0.95149 \\
\hline $\mathrm{C}$ & 3.101955 & -0.93477 & -0.17666 & $\mathrm{C}$ & -4.83292 & -0.4076 & -0.95457 \\
\hline $\mathrm{C}$ & 2.204499 & -1.98648 & -0.18235 & $\mathrm{C}$ & 0.389906 & 4.911048 & 0.215489 \\
\hline $\mathrm{C}$ & 0.76494 & -1.97356 & -0.16114 & $\mathrm{C}$ & -0.31143 & 5.694223 & -0.71566 \\
\hline $\mathrm{C}$ & -0.06753 & -3.10671 & -0.208 & $\mathrm{H}$ & -0.81197 & 5.203539 & -1.54469 \\
\hline $\mathrm{H}$ & 0.291632 & -4.12254 & -0.26664 & $\mathrm{C}$ & -0.35793 & 7.083628 & -0.58801 \\
\hline $\mathrm{C}$ & -1.39858 & -2.69491 & -0.17554 & $\mathrm{H}$ & -0.90155 & 7.673682 & -1.3202 \\
\hline $\mathrm{H}$ & -2.27621 & -3.32313 & -0.19643 & $\mathrm{C}$ & 0.298957 & 7.712671 & 0.471907 \\
\hline $\mathrm{C}$ & -1.40749 & -1.29883 & -0.1015 & $\mathrm{H}$ & 0.265391 & 8.793653 & 0.570733 \\
\hline $\mathrm{C}$ & -2.49458 & -0.37072 & -0.03895 & $\mathrm{C}$ & 1.001811 & 6.94356 & 1.404259 \\
\hline $\mathrm{C}$ & -2.22259 & 0.9812 & 0.000955 & $\mathrm{H}$ & 1.510396 & 7.424791 & 2.234626 \\
\hline $\mathrm{C}$ & -3.02739 & 2.167188 & 0.104516 & $\mathrm{C}$ & 1.047038 & 5.555649 & 1.277488 \\
\hline $\mathrm{H}$ & -4.10693 & 2.174475 & 0.142025 & $\mathrm{H}$ & 1.586817 & 4.958189 & 2.005453 \\
\hline $\mathrm{C}$ & -2.18966 & 3.248985 & 0.15325 & & & & \\
\hline
\end{tabular}


Table S6. $\mathrm{S}_{0}$ optimized geometry of the compound 10.F- at B3LYP/6-31g $(\mathrm{d}, \mathrm{p})$ level of theory \# Sum of imaginary frequencies $=0$ \# Total Energy $($ hartree $)=-2666.906308$

\begin{tabular}{|c|c|c|c|c|c|c|c|}
\hline Atom & $\mathbf{X}$ & $\mathbf{Y}$ & $\mathbf{Z}$ & Atom & $\mathbf{X}$ & $\mathbf{Y}$ & $\mathbf{Z}$ \\
\hline $\mathrm{N}$ & -0.10684 & -0.86458 & 0.024143 & $\mathrm{H}$ & -2.37533 & 4.371174 & 0.166747 \\
\hline $\mathrm{N}$ & -0.87696 & 1.428598 & 0.170232 & $\mathrm{C}$ & -0.76983 & 2.798292 & 0.168495 \\
\hline B & 0.271901 & 0.514326 & 0.591844 & $\mathrm{C}$ & 0.473691 & 3.415547 & 0.133626 \\
\hline $\mathrm{C}$ & 1.628264 & 1.180903 & 0.08549 & $\mathrm{C}$ & 2.622193 & -3.43502 & -0.21742 \\
\hline $\mathrm{C}$ & 1.688582 & 2.617477 & -0.00125 & $\mathrm{C}$ & 2.95696 & -4.15087 & -1.33467 \\
\hline $\mathrm{C}$ & 2.930923 & 3.253023 & -0.27567 & $\mathrm{H}$ & 2.895928 & -3.72381 & -2.32906 \\
\hline $\mathrm{H}$ & 2.966179 & 4.331726 & -0.37538 & $\mathrm{C}$ & 3.417452 & -5.47393 & -1.04943 \\
\hline $\mathrm{C}$ & 2.805544 & 0.458814 & -0.12144 & $\mathrm{H}$ & 3.735167 & -6.18077 & -1.80863 \\
\hline $\mathrm{C}$ & 4.044022 & 1.126442 & -0.33334 & $\mathrm{C}$ & 3.416897 & -5.75718 & 0.28958 \\
\hline $\mathrm{C}$ & 4.096988 & 2.519265 & -0.41991 & $\mathrm{H}$ & 3.708701 & -6.67621 & 0.780544 \\
\hline $\mathrm{H}$ & 5.043215 & 3.022989 & -0.60599 & S & 2.840141 & -4.40815 & 1.220765 \\
\hline $\mathrm{C}$ & 6.475843 & 0.325795 & -0.53524 & $\mathrm{~F}$ & -3.56817 & -2.3018 & 1.739854 \\
\hline $\mathrm{H}$ & 6.880783 & 1.327776 & -0.65496 & $\mathrm{~F}$ & -6.10325 & -3.18696 & 1.615399 \\
\hline $\mathrm{C}$ & 7.31324 & -0.78445 & -0.51946 & $\mathrm{~F}$ & -7.85351 & -2.12092 & -0.19481 \\
\hline $\mathrm{H}$ & 8.387629 & -0.66155 & -0.63097 & $\mathrm{~F}$ & -7.00535 & -0.17039 & -1.91394 \\
\hline $\mathrm{C}$ & 6.766786 & -2.06813 & -0.34944 & $\mathrm{~F}$ & -4.4601 & 0.680211 & -1.859 \\
\hline $\mathrm{H}$ & 7.424283 & -2.9334 & -0.32486 & $\mathrm{C}$ & -3.89893 & -0.76592 & -0.0481 \\
\hline $\mathrm{C}$ & 5.095505 & 0.14448 & -0.38487 & $\mathrm{C}$ & -4.37704 & -1.76803 & 0.812994 \\
\hline $\mathrm{C}$ & 4.520035 & -1.15368 & -0.22822 & $\mathrm{C}$ & -5.68782 & -2.23411 & 0.76523 \\
\hline $\mathrm{C}$ & 5.392728 & -2.25999 & -0.2071 & $\mathrm{C}$ & -6.58441 & -1.68857 & -0.14964 \\
\hline $\mathrm{H}$ & 5.030588 & -3.26893 & -0.07954 & $\mathrm{C}$ & -6.15176 & -0.68844 & -1.0152 \\
\hline $\mathrm{C}$ & 3.053738 & -1.00394 & -0.11361 & $\mathrm{C}$ & -4.82982 & -0.25462 & -0.96621 \\
\hline $\mathrm{C}$ & 2.122726 & -2.0357 & -0.13299 & $\mathrm{C}$ & 0.525068 & 4.896606 & 0.184359 \\
\hline $\mathrm{C}$ & 0.690463 & -1.97912 & -0.14859 & $\mathrm{C}$ & -0.22028 & 5.687568 & -0.70782 \\
\hline $\mathrm{C}$ & -0.16748 & -3.08492 & -0.39153 & $\mathrm{H}$ & -0.81054 & 5.195351 & -1.47412 \\
\hline $\mathrm{H}$ & 0.169503 & -4.09022 & -0.59602 & $\mathrm{C}$ & -0.19907 & 7.081426 & -0.6252 \\
\hline $\mathrm{C}$ & -1.47633 & -2.62991 & -0.36316 & $\mathrm{H}$ & -0.78265 & 7.670709 & -1.32776 \\
\hline $\mathrm{H}$ & -2.37723 & -3.20242 & -0.5339 & $\mathrm{C}$ & 0.578274 & 7.716703 & 0.345697 \\
\hline $\mathrm{C}$ & -1.43059 & -1.24404 & -0.0929 & $\mathrm{H}$ & 0.598758 & 8.801238 & 0.408713 \\
\hline $\mathrm{C}$ & -2.50445 & -0.2996 & 0.006572 & $\mathrm{C}$ & 1.335417 & 6.944069 & 1.23272 \\
\hline $\mathrm{C}$ & -2.19291 & 1.052576 & 0.103771 & $\mathrm{H}$ & 1.942771 & 7.427192 & 1.993616 \\
\hline $\mathrm{C}$ & -2.9847 & 2.259752 & 0.117112 & $\mathrm{C}$ & 1.309183 & 5.552669 & 1.151819 \\
\hline $\mathrm{H}$ & -4.06307 & 2.297766 & 0.057081 & $\mathrm{H}$ & 1.893651 & 4.952522 & 1.842037 \\
\hline $\mathrm{C}$ & -2.12059 & 3.321149 & 0.164693 & $\mathrm{~F}$ & 0.304755 & 0.410427 & 2.019364 \\
\hline
\end{tabular}


Table S7. $\mathrm{S}_{0}$ optimized geometry of the compound 10.CN- at B3LYP/6-31g (d,p) level of theory \# Sum of imaginary frequencies $=0$ \# Total Energy $($ hartree $)=-2659.870457$

\begin{tabular}{|c|c|c|c|c|c|c|c|}
\hline Atom & $\mathbf{X}$ & $\mathbf{Y}$ & $\mathbf{Z}$ & Atom & $\mathbf{X}$ & $\mathbf{Y}$ & $\mathbf{Z}$ \\
\hline $\mathrm{N}$ & -0.11354 & -0.86724 & -0.07263 & $\mathrm{C}$ & -0.76585 & 2.805055 & 0.046036 \\
\hline $\mathrm{N}$ & -0.87789 & 1.433536 & 0.020168 & $\mathrm{C}$ & 0.479726 & 3.414592 & 0.051943 \\
\hline B & 0.251614 & 0.518184 & 0.479723 & $\mathrm{C}$ & 2.608545 & -3.4518 & -0.24285 \\
\hline $\mathrm{C}$ & 1.628925 & 1.173322 & 0.008464 & $\mathrm{C}$ & 2.933172 & -4.19074 & -1.34826 \\
\hline $\mathrm{C}$ & 1.692783 & 2.608467 & -0.08061 & $\mathrm{H}$ & 2.866014 & -3.78399 & -2.35074 \\
\hline $\mathrm{C}$ & 2.935073 & 3.236861 & -0.358 & $\mathrm{C}$ & 3.392178 & -5.50876 & -1.03895 \\
\hline $\mathrm{H}$ & 2.972926 & 4.314992 & -0.46168 & $\mathrm{H}$ & 3.702753 & -6.23214 & -1.78536 \\
\hline $\mathrm{C}$ & 2.800404 & 0.442563 & -0.19218 & $\mathrm{C}$ & 3.399956 & -5.76375 & 0.305764 \\
\hline $\mathrm{C}$ & 4.041459 & 1.107104 & -0.40647 & $\mathrm{H}$ & 3.692302 & -6.67323 & 0.813886 \\
\hline $\mathrm{C}$ & 4.099638 & 2.497532 & -0.49877 & S & 2.833589 & -4.39474 & 1.213298 \\
\hline $\mathrm{H}$ & 5.047775 & 2.99728 & -0.68428 & $\mathrm{~F}$ & -3.5377 & -2.27483 & 1.683412 \\
\hline $\mathrm{C}$ & 6.470746 & 0.299339 & -0.60518 & $\mathrm{~F}$ & -6.07455 & -3.16138 & 1.622092 \\
\hline $\mathrm{H}$ & 6.877837 & 1.299435 & -0.73199 & $\mathrm{~F}$ & -7.86246 & -2.11612 & -0.16243 \\
\hline $\mathrm{C}$ & 7.305081 & -0.81312 & -0.58473 & $\mathrm{~F}$ & -7.0515 & -0.18525 & -1.92121 \\
\hline $\mathrm{H}$ & 8.379427 & -0.69383 & -0.69924 & $\mathrm{~F}$ & -4.50571 & 0.667233 & -1.92804 \\
\hline $\mathrm{C}$ & 6.755593 & -2.09421 & -0.40661 & $\mathrm{C}$ & -3.90687 & -0.75977 & -0.11443 \\
\hline $\mathrm{H}$ & 7.41096 & -2.96087 & -0.37894 & $\mathrm{C}$ & -4.36598 & -1.75201 & 0.768523 \\
\hline $\mathrm{C}$ & 5.090774 & 0.121505 & -0.45116 & $\mathrm{C}$ & -5.67747 & -2.21836 & 0.75323 \\
\hline $\mathrm{C}$ & 4.512663 & -1.17321 & -0.28522 & $\mathrm{C}$ & -6.5932 & -1.68327 & -0.14885 \\
\hline $\mathrm{C}$ & 5.381577 & -2.28223 & -0.26 & $\mathrm{C}$ & -6.17935 & -0.69312 & -1.03487 \\
\hline $\mathrm{H}$ & 5.016757 & -3.28932 & -0.12674 & $\mathrm{C}$ & -4.85696 & -0.25876 & -1.01828 \\
\hline $\mathrm{C}$ & 3.046341 & -1.02046 & -0.17342 & $\mathrm{C}$ & 0.545468 & 4.892681 & 0.136842 \\
\hline $\mathrm{C}$ & 2.114356 & -2.04959 & -0.18557 & $\mathrm{C}$ & -0.18554 & 5.711766 & -0.74142 \\
\hline $\mathrm{C}$ & 0.681332 & -1.98681 & -0.22047 & $\mathrm{H}$ & -0.77553 & 5.24449 & -1.52339 \\
\hline $\mathrm{C}$ & -0.17892 & -3.08849 & -0.45993 & $\mathrm{C}$ & -0.14878 & 7.10288 & -0.6258 \\
\hline $\mathrm{H}$ & 0.15563 & -4.09677 & -0.65269 & $\mathrm{H}$ & -0.72088 & 7.715328 & -1.31778 \\
\hline $\mathrm{C}$ & -1.48778 & -2.62712 & -0.44754 & $\mathrm{C}$ & 0.62938 & 7.705647 & 0.364942 \\
\hline $\mathrm{H}$ & -2.38933 & -3.19726 & -0.62189 & $\mathrm{H}$ & 0.661529 & 8.788023 & 0.453917 \\
\hline $\mathrm{C}$ & -1.43972 & -1.24191 & -0.18966 & $\mathrm{C}$ & 1.372265 & 6.904033 & 1.238246 \\
\hline $\mathrm{C}$ & -2.51222 & -0.29243 & -0.09327 & $\mathrm{H}$ & 1.979692 & 7.362175 & 2.014216 \\
\hline $\mathrm{C}$ & -2.19656 & 1.060485 & -0.0301 & $\mathrm{C}$ & 1.33168 & 5.515467 & 1.124265 \\
\hline $\mathrm{C}$ & -2.98148 & 2.271047 & -0.01386 & $\mathrm{H}$ & 1.90447 & 4.892466 & 1.803808 \\
\hline $\mathrm{H}$ & -4.05935 & 2.313746 & -0.07526 & $\mathrm{C}$ & 0.247904 & 0.444292 & 2.117533 \\
\hline $\mathrm{C}$ & -2.11436 & 3.329647 & 0.042091 & $\mathrm{~N}$ & 0.262718 & 0.406736 & 3.282051 \\
\hline $\mathrm{H}$ & -2.36617 & 4.380168 & 0.052565 & & & & \\
\hline
\end{tabular}


Table S8. Selected TD-DFT calculated oscillator strengths and compositions of the major electronic transitions of $\mathbf{1 0}$

\begin{tabular}{|c|c|c|}
\hline $\begin{array}{l}\text { Wavelength } \\
\text { (nm) }\end{array}$ & $\begin{array}{l}\text { Oscillator } \\
\text { Strength }\end{array}$ & Major contributions \\
\hline 736.2868142 & 0.0631 & HOMO->LUMO (96\%) \\
\hline 509.2345531 & 0.1074 & H-1->LUMO (33\%), HOMO->L+1 (62\%) \\
\hline 435.2888974 & 0.7207 & H-1->LUMO (54\%), HOMO->L+1 (30\%) \\
\hline 391.5345691 & 0.1147 & H-1->L+1 (42\%), HOMO->L+2 (51\%) \\
\hline 380.3169836 & 0.344 & $\begin{array}{l}\mathrm{H}-2->\mathrm{LUMO}(28 \%), \mathrm{H}-1->\mathrm{L}+1 \text { (29\%), HOMO- } \\
>\mathrm{L}+2(31 \%)\end{array}$ \\
\hline 365.0218944 & 0.0199 & H-3->LUMO (78\%) \\
\hline 360.5634172 & 0.1464 & H-2->LUMO (11\%), HOMO->L+3 (70\%) \\
\hline 356.2330095 & 0.4953 & $\begin{array}{l}\text { H-2->LUMO (43\%), H-1->L+1 (18\%), HOMO- } \\
>\mathrm{L}+3(17 \%)\end{array}$ \\
\hline 337.1861209 & 0.0009 & HOMO->L+4 (38\%), HOMO->L+5 (61\%) \\
\hline 336.2351159 & 0.0003 & HOMO->L+4 (60\%), HOMO->L+5 (38\%) \\
\hline 333.4498861 & 0.0394 & H-4->LUMO (93\%) \\
\hline 319.5446821 & 0.0576 & H-5->LUMO (61\%), HOMO->L+6 (17\%) \\
\hline 317.580268 & 0.0157 & $\begin{array}{l}\mathrm{H}-3->\mathrm{L}+1(45 \%), \mathrm{H}-2->\mathrm{L}+1(24 \%), \mathrm{HOMO}->\mathrm{L}+6 \\
(19 \%)\end{array}$ \\
\hline 315.9616122 & 0.0152 & $\begin{array}{l}\text { H-5->LUMO (10\%), H-3->L+1 (29\%), HOMO- } \\
>\mathrm{L}+6(39 \%)\end{array}$ \\
\hline 313.659524 & 0.0277 & $\begin{array}{l}\text { H-6->LUMO (36\%), H-2->L+1 (15\%), HOMO- } \\
>\text { L+7 (20\%) }\end{array}$ \\
\hline 311.0313999 & 0.0398 & $\begin{array}{l}\text { H-6->LUMO (48\%), H-2->L+1 (19\%), HOMO- } \\
>\mathrm{L}+8(12 \%)\end{array}$ \\
\hline 309.9660908 & 0.05 & H-7->LUMO (35\%), HOMO->L+7 (33\%) \\
\hline 308.0331345 & 0.041 & HOMO- $>$ L $+8(51 \%)$ \\
\hline 306.0032496 & 0.0214 & H-7->LUMO (40\%), HOMO->L+7 (31\%) \\
\hline 303.1599791 & 0.0216 & H-8->LUMO (86\%) \\
\hline 299.5707267 & 0.2231 & H-7->LUMO (13\%), H-1->L+2 (58\%) \\
\hline 295.5502661 & 0.0903 & $\begin{array}{l}\text { H-11->LUMO (27\%), H-10->LUMO (16\%), H-9- } \\
\text { >LUMO }(36 \%)\end{array}$ \\
\hline 291.9521903 & 0.0926 & H-10->LUMO (73\%), H-9->LUMO (10\%) \\
\hline 291.389543 & 0.024 & $\begin{array}{l}\text { H-11->LUMO (13\%), H-4->L+1 (52\%), H-1- } \\
>\mathrm{L}+3(14 \%)\end{array}$ \\
\hline 287.9917694 & 0.0064 & $\begin{array}{l}\text { H-11->LUMO (42\%), H-9->LUMO (27\%), H-4- } \\
>\text { L+1 }(10 \%)\end{array}$ \\
\hline 281.8828134 & 0.1364 & H-4->L+1 (16\%), H-1->L+3 (66\%) \\
\hline 275.8495453 & 0.0021 & HOMO->L+9 (99\%) \\
\hline 271.1440682 & 0.0122 & $\mathrm{H}-1->\mathrm{L}+5(91 \%)$ \\
\hline 270.5110655 & 0.3773 & $\mathrm{H}-5->\mathrm{L}+1(68 \%), \mathrm{H}-2->\mathrm{L}+2(11 \%)$ \\
\hline
\end{tabular}




\begin{tabular}{|c|c|c|}
\hline 268.455172 & 0.0676 & H-7->L+1 (37\%), H-6->L+1 (40\%) \\
\hline 266.1957588 & 0.001 & $\mathrm{H}-1->\mathrm{L}+4(95 \%)$ \\
\hline 262.6042332 & 0.0361 & H-1->L+8 (19\%), HOMO->L+10 (44\%) \\
\hline 261.0890068 & 0.0029 & $\begin{array}{l}\text { H-7->L+1 }(21 \%), \text { H-6->L+1 }(35 \%), H-2->L+2 \\
(10 \%), \text { HOMO->L+10 }(15 \%)\end{array}$ \\
\hline 260.4856118 & 0.0103 & $\begin{array}{l}\text { H-12->LUMO (10\%), H-9->L+1 (21\%), H-7- } \\
>\mathrm{L}+1(13 \%), \mathrm{H}-6->\mathrm{L}+1(11 \%), \mathrm{H}-2->\mathrm{L}+2(25 \%)\end{array}$ \\
\hline 258.0352071 & 0.0099 & $\mathrm{H}-8->\mathrm{L}+1(81 \%)$ \\
\hline 255.6410166 & 0.1484 & $\begin{array}{l}\text { H-12->LUMO (19\%), H-9->L+1 (18\%), H-1- } \\
>\text { L+6 (31\%) }\end{array}$ \\
\hline 255.0520183 & 0.0085 & $\begin{array}{l}\text { H-10->L+1 (16\%), H-9->L+1 (25\%), H-1->L+6 } \\
(21 \%)\end{array}$ \\
\hline 252.965267 & 0.2463 & $\begin{array}{l}\text { H-12->LUMO (29\%), H-11->L+1 (10\%), H-2- } \\
>\text { L+2 (16\%), H-1->L+6 }(32 \%)\end{array}$ \\
\hline 251.1868892 & 0.002 & H-11->L+1 (10\%), HOMO->L+11 (64\%) \\
\hline 250.5574372 & 0.0304 & $\begin{array}{l}\mathrm{H}-10->\mathrm{L}+1(44 \%), \mathrm{H}-2->\mathrm{L}+2(13 \%), \mathrm{HOMO}- \\
>\mathrm{L}+11(12 \%)\end{array}$ \\
\hline 249.7851089 & 0.0107 & $\mathrm{H}-3->\mathrm{L}+2(70 \%)$ \\
\hline 249.6794745 & 0.0404 & $\begin{array}{l}\mathrm{H}-3->\mathrm{L}+2(15 \%), \mathrm{H}-1->\mathrm{L}+7(62 \%), \mathrm{H}-1->\mathrm{L}+8 \\
(10 \%)\end{array}$ \\
\hline 249.0625485 & 0.0491 & $\begin{array}{l}\text { H-11->L+1 (26\%), H-9->L+1 (11\%), H-1->L+8 } \\
(18 \%), \text { HOMO->L+11 (16\%) }\end{array}$ \\
\hline 246.7919436 & 0.0796 & $\begin{array}{l}\mathrm{H}-11->\mathrm{L}+1(22 \%), \mathrm{H}-1->\mathrm{L}+8(25 \%), \mathrm{HOMO}- \\
>\mathrm{L}+12(30 \%)\end{array}$ \\
\hline 243.8121148 & 0.1362 & $\mathrm{H}-2->\mathrm{L}+3(73 \%)$ \\
\hline 241.7536056 & 0.1043 & $\begin{array}{l}\text { H-13->LUMO (37\%), H-4->L+2 (20\%), HOMO- } \\
>\mathrm{L}+12(23 \%)\end{array}$ \\
\hline 239.5350399 & 0.0591 & H-13->LUMO (31\%), H-4->L+2 (47\%) \\
\hline 237.4204566 & 0.0027 & $\mathrm{H}-3->\mathrm{L}+3(94 \%)$ \\
\hline 236.4109081 & 0.0015 & $\begin{array}{l}\mathrm{H}-10->\mathrm{L}+4(10 \%), \mathrm{H}-8->\mathrm{L}+2(18 \%), \mathrm{H}-8->\mathrm{L}+3 \\
(20 \%), \mathrm{H}-2->\mathrm{L}+4(37 \%)\end{array}$ \\
\hline 235.2892865 & 0.0217 & $\begin{array}{l}\text { H-13->LUMO (20\%), H-2->L+5 (12\%), HOMO- } \\
>\text { L+12 (20\%) }\end{array}$ \\
\hline
\end{tabular}


Table S9. Selected TD-DFT calculated oscillator strengths and compositions of the major electronic transitions of $\mathbf{1 0 . F ^ { - }}$

\begin{tabular}{|c|r|l|l|}
\hline $\begin{array}{l}\text { Wavelength } \\
\text { (nm) }\end{array}$ & $\begin{array}{l}\text { Oscillator } \\
\text { Strength }\end{array}$ & \multicolumn{1}{|c|}{ Major contribs } & \multicolumn{1}{|c|}{ Minor contribs } \\
\hline 724.07485 & 0.1348 & $\mathrm{H}->\mathrm{L}(93 \%)$ & $\mathrm{H}->\mathrm{L}+1(5 \%)$ \\
\hline 553.101966 & 0.2612 & $\mathrm{H}-1->\mathrm{L}(16 \%), \mathrm{H}->\mathrm{L}+1(78 \%)$ & $\mathrm{H}->\mathrm{L}(4 \%)$ \\
\hline 441.111953 & 0.6474 & $\mathrm{H}-1->\mathrm{L}(49 \%), \mathrm{H}->\mathrm{L}+2(40 \%)$ & $\mathrm{H}->\mathrm{L}+1(8 \%)$ \\
\hline 417.663253 & 0.154 & $\begin{array}{l}\mathrm{H}-1->\mathrm{L}(15 \%), \mathrm{H}->\mathrm{L}+2(37 \%), \mathrm{H}->\mathrm{L}+3 \\
(14 \%), \mathrm{HOMO}-\mathrm{L}+4(23 \%)\end{array}$ & $\begin{array}{l}\mathrm{H}-1->\mathrm{L}+1(5 \%), \mathrm{H}- \\
>\mathrm{L}+1(3 \%)\end{array}$ \\
\hline 413.512112 & 0.0061 & $\mathrm{H}->\mathrm{L}+3(78 \%), \mathrm{H}->\mathrm{L}+4(18 \%)$ & \\
\hline 409.780991 & 0.1158 & $\begin{array}{l}\mathrm{H}-1->\mathrm{L}(10 \%), \mathrm{H}-1->\mathrm{L}+1(17 \%), \mathrm{H}- \\
>\mathrm{L}+4(54 \%)\end{array}$ & $\begin{array}{l}\mathrm{H}->\mathrm{L}+2(9 \%), \mathrm{H}->\mathrm{L}+3 \\
(5 \%)\end{array}$ \\
\hline
\end{tabular}

Table S10. Selected TD-DFT calculated oscillator strengths and compositions of the major electronic transitions of $\mathbf{1 0 . C N}$.

\begin{tabular}{|c|c|c|c|}
\hline $\begin{array}{l}\text { Wavelength } \\
\text { (nm) }\end{array}$ & $\begin{array}{l}\text { Oscillator } \\
\text { Strength }\end{array}$ & Major contribs & Minor contribs \\
\hline 744.4211 & 0.1249 & $\mathrm{H}->\mathrm{L}(94 \%)$ & $\mathrm{H}->\mathrm{L}+1(4 \%)$ \\
\hline 556.1287 & 0.2408 & H-1->L (18\%), H->L+1 (76\%) & $\mathrm{H}->\mathrm{L}(3 \%)$ \\
\hline 443.4628 & 0.8539 & $\begin{array}{l}\mathrm{H}-1->\mathrm{L}(64 \%), \mathrm{H}->\mathrm{L}+1(13 \%), \mathrm{H}->\mathrm{L}+2 \\
(19 \%)\end{array}$ & $\mathrm{H}->\mathrm{L}(3 \%)$ \\
\hline 413.3605 & 0.078 & $\mathrm{H}-1->\mathrm{L}+1(18 \%), \mathrm{H}->\mathrm{L}+2(62 \%)$ & $\begin{array}{l}\mathrm{H}-2->\mathrm{L}(5 \%), \mathrm{H}-1- \\
>\mathrm{L}(9 \%)\end{array}$ \\
\hline 402.6087 & 0.0116 & $\begin{array}{l}\mathrm{H}-1->\mathrm{L}+1(11 \%), \mathrm{H}->\mathrm{L}+3(64 \%), \mathrm{H}- \\
>\mathrm{L}+4(20 \%)\end{array}$ & $\mathrm{H}-2->\mathrm{L}(3 \%)$ \\
\hline 401.5915 & 0.0031 & $\mathrm{H}->\mathrm{L}+3(30 \%), \mathrm{H}->\mathrm{L}+4(66 \%)$ & $\mathrm{H}-1->\mathrm{L}+1(3 \%)$ \\
\hline
\end{tabular}




\section{References}

1. Frisch, M. J.; Trucks, G. W.; Schlegel, H. B.; Scuseria, G. E.; Robb, M. A.; Cheeseman, J. R.; Scalmani, G.; Barone, V.; Mennucci, B.; Petersson, G. A.; et al. Gaussian 09 C.01; Gaussian Inc.: Wallingford CT, 2009.

2. Treutler, O.; Ahlrichs, R. Efficient Molecular Numerical Integration Schemes. J. Chem. Phys. 1995, 102, 346-354.

3. Becke, A. D. A New Mixing of Hartree-Fock and Local Density-Functional Theories. $J$. Chem. Phys. 1993, 98, 1372-1377.

4. Hehre, W. J. Ab Initio Molecular Orbital Theory. Acc. Chem. Res. 1976, 9, 399-406.

5. Tomasi, J.; Mennucci, B.; Cammi, R. Quantum Mechanical Continuum Solvation Models. Chem. Rev. 2005, 105, 2999-3093. 